\title{
Probabilistic positional association of catalogs of astrophysical sources: the ASPECTS code ${ }^{\star, \star \star}$
}

\author{
Michel Fioc
}

\author{
Institut d'Astrophysique de Paris, UPMC - Univ. Paris 6, CNRS, UMR 7095, 98bis boulevard Arago, 75014 Paris, France \\ e-mail: Michel.Fioc@iap.fr
}

Received 16 July 2012 / Accepted 1 November 2013

\begin{abstract}
We describe a probabilistic method of cross-identifying astrophysical sources in two catalogs from their positions and positional uncertainties. The probability that an object is associated with a source from the other catalog, or that it has no counterpart, is derived under two exclusive assumptions: first, the classical case of several-to-one associations, and then the more realistic but more difficult problem of one-to-one associations. In either case, the likelihood of observing the objects in the two catalogs at their effective positions is computed and a maximum likelihood estimator of the fraction of sources with a counterpart - a quantity needed to compute the probabilities of association - is built. When the positional uncertainty in one or both catalogs is unknown, this method may be used to estimate its typical value and even to study its dependence on the size of objects. It may also be applied when the true centers of a source and of its counterpart at another wavelength do not coincide. To compute the likelihood and association probabilities under the different assumptions, we developed a Fortran 95 code called AsPECTs ([aspe], "Association PositionnellE/ProbabilistE de CaTalogues de Sources" in French); its source files are made freely available. To test ASPECTs, all-sky mock catalogs containing up to $10^{5}$ objects were created, forcing either several-to-one or one-to-one associations. The analysis of these simulations confirms that, in both cases, the assumption with the highest likelihood is the right one and that estimators of unknown parameters built for the appropriate association model are reliable.
\end{abstract}

Key words. methods: statistical - catalogs - galaxies: statistics - stars: statistics - astrometry

\section{Introduction}

The most basic method of cross-identifying two catalogs $K$ and $K^{\prime}$ with known circular positional uncertainties is to consider that a $K^{\prime}$-source $M^{\prime}$ is the same as an object $M$ of $K$ if it falls within a disk centered on $M$ and having a radius equal to a few times their combined positional uncertainty; if the disk is void, $M$ has no counterpart, and if it contains several $K^{\prime}$-sources, the nearest one is identified to $M$. This solution is defective for several reasons: it does not take the density of sources into account; positional uncertainty ellipses are not properly treated; the radius of the disk is arbitrary; positional uncertainties are not always known; $K$ and $K^{\prime}$ do not play symmetrical roles; the identification is ambiguous if a $K^{\prime}$-source may be associated to several objects of $K$. Worst of all, it does not provide a probability of association.

Beyond this naïve method, the cross-identification problem has been studied by Condon et al. (1975), de Ruiter et al. (1977), Prestage \& Peacock (1983), Sutherland \& Saunders (1992), Bauer et al. (2000), and Rutledge et al. (2000), among others. As shown by the recent papers of Budavári \& Szalay (2008), Brand et al. (2006), Rohde et al. (2006), Roseboom et al. (2009), and Pineau et al. (2011), this field is still very active and will be more so with the wealth of forthcoming multiwavelength data and the virtual observatory (Vignali et al. 2009). In these papers,

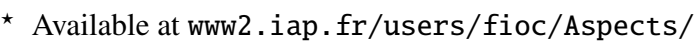

$\star \star$ The AsPECTS code is available at the CDS via anonymous ftp to cdsarc.u-strasbg. fr (130.79.128.5) or via

http://cdsarc.u-strasbg.fr/viz-bin/qcat?]/A+A/566/A8
}

the identification is performed using a "likelihood ratio". For two objects $\left(M, M^{\prime}\right) \in K \times K^{\prime}$ with known coordinates and positional uncertainties, and given the local surface density of $K^{\prime}$-sources, this ratio is typically computed as

$\lambda:=\frac{P(\text { position } \mid \text { counterpart })}{P(\text { position } \mid \text { chance })}$,

where $P$ (position | counterpart) is the probability of finding $M^{\prime}$ at some position relative to $M$ if $M^{\prime}$ is a counterpart of $M$, and $P$ (position | chance) is the probability that $M^{\prime}$ is there by chance. As noticed by Sutherland \& Saunders (1992), there has been some confusion when defining and interpreting $\lambda$, and, more importantly, in deriving the probability ${ }^{1}$ that $M$ and $M^{\prime}$ are the same.

To associate sources from catalogs at different wavelengths, some authors include some a priori information on the spectral energy distribution (SED) of the objects in this likelihood ratio. When this work started, our primary goal was to build template observational SEDs from the optical to the far-infrared for different types of galaxies. We initially intended to cross-identify the IRAS Faint Source Survey (Moshir et al. 1992, 1993) with the LEDA database (Paturel et al. 1995). Because of the high positional inaccuracy of IRAS data, special care was needed to identify optical sources with infrared ones. While IRAS data are by now quite outdated and have been superseded by Spitzer and Herschel observations, we think that the procedure we began to

\footnotetext{
1 For instance, de Ruiter et al. (1977) wrongly state that, if there is a counterpart, the closest object is always the right one.
} 
develop at that time may be valuable for other studies. Because we aimed to fit synthetic SEDs to the template observational ones, we could not and did not want to make assumptions on the SED of objects based on their type, since this would have biased the procedure. We therefore rely only on positions in what follows.

The method we use is in essence similar to that of Sutherland $\&$ Saunders (1992). Because thinking in terms of probabilities rather than of likelihood ratios highlights some implicit assumptions, we found it however useful for the sake of clarity to detail hereafter our calculations. This allows us moreover to propose a systematic way to estimate the unknown parameters required to compute the probabilities of association and to extend our work to a case not covered by the papers cited above (see Sect. 4).

After some preliminaries (Sect. 2), we compute in Sect. 3 the probability of association under the hypothesis that a $K$-source has at most one counterpart in $K^{\prime}$ but that several objects of $K$ may share the same one ("several-to-one" associations). We also compute the likelihood to observe all the sources at their effective positions and use it to estimate the fraction of objects with a counterpart and, if unknown, the positional uncertainty in one or both catalogs. In Sect. 4, we do the same calculations under the assumption that a $K$-source has at most one counterpart in $K^{\prime}$ and that no other object of $K$ has the same counterpart ("oneto-one" associations). In Sect. 5, we present a code, AsPECTs, implementing the results of Sects. 3 and 4, and with which we compute the likelihoods and probabilities of association under the aforementioned assumptions. We test it on simulations in Sect. 6. The probability distribution of the relative positions of associated sources is modeled in Appendix A.

\section{Preliminaries}

\subsection{Notations}

We consider two catalogs $K$ and $K^{\prime}$ defined on a common surface of the sky, of area $S$, and containing respectively $n$ sources $\left(M_{i}\right)_{i \in \llbracket 1, n \rrbracket}$ and $n^{\prime}$ sources $\left(M_{j}^{\prime}\right)_{j \in \llbracket 1, n^{\prime} \rrbracket}$. We define the following events:

$-c_{i}: M_{i}$ is in the infinitesimal surface element $\mathrm{d}^{2} \boldsymbol{r}_{i}$ located at $\boldsymbol{r}_{i}$;

- $c_{j}^{\prime}: M_{j}^{\prime}$ is in the infinitesimal surface element $\mathrm{d}^{2} \boldsymbol{r}_{j}^{\prime}$ located at $\boldsymbol{r}_{j}^{\prime}$;

- $C:=\bigcap_{i=1}^{n} c_{i}$ : the coordinates of all $K$-sources are known;

- $C^{\prime}:=\bigcap_{j=1}^{n^{\prime}} c_{j}^{\prime}$ : the coordinates of all $K^{\prime}$-sources are known;

- $A_{i, j}$, with $i \neq 0$ and $j \neq 0: M_{j}^{\prime}$ is a counterpart of $M_{i}$;

- $A_{i, 0}: M_{i}$ has no counterpart in $K^{\prime}$, i.e. $A_{i, 0}=\overline{\bigcup_{j \neq 0} A_{i, j}}$, where $\bar{\omega}$ is the negation of an event $\omega$;

- $A_{0, j}: M_{j}^{\prime}$ has no counterpart in $K$.

We denote by $f$ (resp. $f^{\prime}$ ) the unknown a priori (i.e., not knowing the coordinates) probability that any element of $K$ (resp. $K^{\prime}$ ) has a counterpart in $K^{\prime}$ (resp. $K$ ). In terms of the events $\left(A_{i, j}\right)$, for any $\left(M_{i}, M_{j}^{\prime}\right) \in K \times K^{\prime}$,

$$
\left.\begin{array}{l}
P\left(\bigcup_{k \neq 0} A_{i, k}\right)=f ; \quad P\left(A_{i, 0}\right)=1-f ; \\
P\left(\bigcup_{k \neq 0} A_{k, j}\right)=f^{\prime} ; \quad P\left(A_{0, j}\right)=1-f^{\prime} .
\end{array}\right\}
$$

We see in Sects. 3.2 and 4.2 how to estimate $f$ and $f^{\prime}$.

The angular distance between two points $Y$ and $Z$ is written $\psi(Y, Z)$. More specifically, we put $\psi_{i, j}=\psi\left(M_{i}, M_{j}^{\prime}\right)$.

\subsection{Assumptions}

Calculations are carried out under one of three exclusive assumptions:

\section{- Several-to-one hypothesis:}

$\left\{\right.$ for all $M_{i}$, the events $\left(A_{i, j}\right)_{j \in \llbracket 1, n^{\prime} \rrbracket}$ are exclusive; $\left\{\right.$ for all $M_{j}^{\prime}$, the events $\left(A_{i, j}\right)_{i \in \llbracket 1, n \rrbracket}$ are independent.

$\left(H_{\mathrm{s}: \mathrm{o}}\right)$

Therefore, a $K$-source has at most one counterpart in $K^{\prime}$, but a $K^{\prime}$-source may have several counterparts in $K$. Since more $K$-sources have a counterpart in $K^{\prime}$ than the converse, $f n \geqslant$ $f^{\prime} n^{\prime}$. This assumption is reasonable if the angular resolution in $K^{\prime}$ (e.g. IRAS) is much poorer than in $K$ (e.g. LEDA), since several distinct objects of $K$ may then be confused in $K^{\prime}$.

- One-to-several hypothesis: the symmetric of assumption $H_{\text {s:o }}$, i.e.,

$\left\{\right.$ for all $M_{i}$, the events $\left(A_{i, j}\right)_{i \in \llbracket 1, n \rrbracket}$ are independent;

$\left(H_{\mathrm{o}: \mathrm{s}}\right)$

for all $M_{j}^{\prime}$, the events $\left(A_{i, j}\right)_{j \in \llbracket 1, n^{\prime} \rrbracket}$ are exclusive.

In that case, $f n \leqslant f^{\prime} n^{\prime}$. This assumption is appropriate for catalogs of extended sources that, although observed as single at the wavelength of $K$, may look broken up at the wavelength of $K^{\prime}$.

- One-to-one hypothesis: any $K$-source has at most one counterpart in $K^{\prime}$ and reciprocally, i.e.

all the events $\left(A_{i, j}\right)_{i \in \llbracket 1, n \rrbracket, j \in \llbracket 1, n^{\prime} \rrbracket}$ are exclusive.

$\left(H_{\mathrm{o:o}}\right)$

Then, $f n=f^{\prime} n^{\prime}$. This assumption is the most relevant one for high-resolution catalogs of point sources or of welldefined extended sources.

Probabilities, likelihoods, and estimators specifically derived under either assumption $H_{\mathrm{s}: 0}, H_{\mathrm{o}: \mathrm{s}}$, or $H_{\mathrm{o}: \mathrm{o}}$ are written with the subscript "s:o", "o:s", or "o:o", respectively; the subscript ":o" is used for results valid for both $H_{\text {s:o }}$ and $H_{\mathrm{o}: 0}$. The "several-toseveral" hypothesis where all the events $\left(A_{i, j}\right)_{i \in \llbracket 1, n \rrbracket, j \in \llbracket 1, n^{\prime} \rrbracket}$ are independent is not considered here.

We make two other assumptions: all the associations $A_{i, j}$ with $i \neq 0$ and $j \neq 0$ are considered a priori as equally likely, and the effect of clustering is negligible.

\subsection{Approach}

Our approach is the following. For each of the assumptions $H_{\mathrm{s}: 0}$, $H_{\text {o:o }}$, and $H_{\text {o:s }}$, we

- find an expression for the probabilities of association,

- build estimators of the unknown parameters needed to compute these probabilities, and

- compute the likelihood of the assumption from the data.

Then, we compute the probabilities of association for the best estimators of unknown parameters and the most likely assumption.

Although $H_{\text {s:o }}$ is less symmetrical and neutral than $H_{\mathrm{o}: o}$, we begin our study with this assumption: first, because computations are much simpler under $H_{\text {s:o }}$ than under $H_{\text {o:o }}$ and serve as a guide for the latter; second, because they provide initial values for the iterative procedure (Sect. 5.4.3) used to effectively compute probabilities under $H_{\mathrm{o}: \mathrm{o}}$.

\section{Several-to-one associations}

In this section, we assume that hypothesis $H_{\text {s:o }}$ holds. As shown in Sect. 3.3, this is also the assumption implicitly made by the authors cited in the introduction. 


\subsection{Probability of association: global computation}

We want to compute ${ }^{2}$ the probability $P\left(A_{i, j} \mid C \cap C^{\prime}\right)$ of association between sources $M_{i}$ and $M_{j}^{\prime}(j \neq 0)$ or the probability that $M_{i}$ has no counterpart $(j=0)$, knowing the coordinates of all the objects in $K$ and $K^{\prime}$. Remembering that, for any events $\omega_{1}, \omega_{2}$, and $\omega_{3}, P\left(\omega_{1} \mid \omega_{2}\right)=P\left(\omega_{1} \cap \omega_{2}\right) / P\left(\omega_{2}\right)$ and thus

$$
\begin{aligned}
& P\left(\omega_{1} \cap \omega_{2} \mid \omega_{3}\right)=\frac{P\left(\omega_{1} \cap \omega_{2} \cap \omega_{3}\right)}{P\left(\omega_{3}\right)} \\
& =\frac{P\left(\omega_{1} \mid \omega_{2} \cap \omega_{3}\right) P\left(\omega_{2} \cap \omega_{3}\right)}{P\left(\omega_{3}\right)}=P\left(\omega_{1} \mid \omega_{2} \cap \omega_{3}\right) P\left(\omega_{2} \mid \omega_{3}\right),
\end{aligned}
$$

we have, with $\omega_{1}=A_{i, j}, \omega_{2}=C$, and $\omega_{3}=C^{\prime}$,

$P\left(A_{i, j} \mid C \cap C^{\prime}\right)=\frac{P\left(A_{i, j} \cap C \mid C^{\prime}\right)}{P\left(C \mid C^{\prime}\right)}$.

\subsubsection{Computation of $P_{\text {s:o }}\left(C \mid C^{\prime}\right)$}

We first compute the denominator of Eq. (4) $)^{3}$. The event

$\bigcap_{k=1}^{n} \bigcup_{j_{k}=0}^{n^{\prime}} A_{k, j_{k}}=\bigcup_{j_{1}=0}^{n^{\prime}} \bigcup_{j_{2}=0}^{n^{\prime}} \cdots \bigcup_{j_{n}=0}^{n^{\prime}} \bigcap_{k=1}^{n} A_{k, j_{k}}$

is certain by definition of the $A_{k, j_{k}}$ and, under either assumption $H_{\text {s:o }}$ or $H_{\mathrm{o}: 0}, A_{k, \ell} \cap A_{k, m} \stackrel{=}{=} \varnothing$ for all $M_{k}$ if $\ell \neq m$. Consequently, using the symbol $\biguplus$ for mutually exclusive events instead of $U$, we obtain

$$
\begin{aligned}
& P_{\text {s:o }}\left(C \mid C^{\prime}\right)=P_{\text {s:o }}\left(C \cap \bigcap_{k=1}^{n} \bigcup_{j_{k}=0}^{n^{\prime}} A_{k, j_{k}} \mid C^{\prime}\right) \\
& =P_{\text {s:o }}\left(C \cap \biguplus \biguplus_{j_{1}=0}^{n^{\prime}}+\cdots \biguplus_{j_{2}=0}^{n^{\prime}} \bigcap_{j_{n}=0}^{n^{\prime}} A_{k=1}\left|C_{k}\right| C^{\prime}\right) \\
& =\sum_{j_{1}=0}^{n^{\prime}} \sum_{j_{2}=0}^{n^{\prime}} \cdots \sum_{j_{n}=0}^{n^{\prime}} P_{\text {s:o }}\left(C \cap \bigcap_{k=1}^{n} A_{k, j_{k}} \mid C^{\prime}\right) \\
& =\sum_{j_{1}=0}^{n^{\prime}} \sum_{j_{2}=0}^{n^{\prime}} \cdots \sum_{j_{n}=0}^{n^{\prime}} P_{\text {s:o }}\left(C \mid \bigcap_{k=1}^{n} A_{k, j_{k}} \cap C^{\prime}\right) P_{\text {s:o }}\left(\bigcap_{k=1}^{n} A_{k, j_{k}} \mid C^{\prime}\right),
\end{aligned}
$$

with $\omega_{1}=C, \omega_{2}=\bigcap_{k=1}^{n} A_{k, j_{k}}$, and $\omega_{3}=C^{\prime}$ in Eq. (3).

Since $C=\bigcap_{k=1}^{n} c_{k}$, the first factor in the product of Eq. (6) is

$$
\begin{aligned}
& P_{: \mathrm{o}}\left(C \mid \bigcap_{k=1}^{n} A_{k, j_{k}} \cap C^{\prime}\right) \\
& =P_{: \mathrm{o}}\left(c_{1} \mid \bigcap_{k=2}^{n} c_{k} \cap \bigcap_{k=1}^{n} A_{k, j_{k}} \cap C^{\prime}\right) P_{: \mathrm{o}}\left(\bigcap_{k=2}^{n} c_{k} \mid \bigcap_{k=1}^{n} A_{k, j_{k}} \cap C^{\prime}\right),
\end{aligned}
$$

\footnotetext{
2 For the sake of clarity, we mention that we adopt the same decreasing order of precedence for operators as in MATHEMATICA (Wolfram 1996): $\times$ and $/ ; \Pi ; \sum ;+$ and - .

3 Computing $P_{\mathrm{s}: \mathrm{o}}\left(C \mid C^{\prime}\right)$ is easier than for $P_{\mathrm{s}: \mathrm{o}}\left(C^{\prime} \mid C\right)$ : the latter would require calculating $P_{\mathrm{s}: \mathrm{o}}\left(c_{\ell}^{\prime} \mid \bigcap_{k=1 ; j_{k}=\ell}^{n}\left[c_{k} \cap A_{k, j_{k}}\right]\right)$ (cf. Eq. (9)) because several $M_{k}$ might be associated with the same $M_{\ell}^{\prime}$. This does not matter for computations made under assumption $H_{\mathrm{o}: 0}$.
}

with $\omega_{1}=c_{1}, \omega_{2}=\bigcap_{k=2}^{n} c_{k}$, and $\omega_{3}=A_{k, j_{k}} \cap C^{\prime}$ in Eq. (3). Doing the same with $\bigcap_{k=2}^{n} c_{k}$ instead of $C$, we obtain

$$
P_{: \mathrm{o}}\left(C \mid \bigcap_{k=1}^{n} A_{k, j_{k}} \cap C^{\prime}\right)=\prod_{\ell=1}^{n} P_{: \mathrm{o}}\left(c_{\ell} \mid \bigcap_{k=\ell+1}^{n} c_{k} \cap \bigcap_{k=1}^{n} A_{k, j_{k}} \cap C^{\prime}\right)
$$

by iteration.

If $j_{\ell} \neq 0, M_{\ell}$ is only associated with $M_{j_{\ell}}^{\prime}$. Consequently,

$$
\begin{aligned}
P_{: \mathrm{o}}\left(c_{\ell} \mid \bigcap_{k=\ell+1}^{n} c_{k} \cap \bigcap_{k=1}^{n} A_{k, j_{k}} \cap C^{\prime}\right) & =P_{: \mathrm{o}}\left(c_{\ell} \mid A_{\ell, j_{\ell}} \cap c_{j_{\ell}}^{\prime}\right) \\
& =\xi_{\ell, j_{\ell}} \mathrm{d}^{2} \boldsymbol{r}_{\ell},
\end{aligned}
$$

where, denoting by $\boldsymbol{r}_{\ell, j_{\ell}}:=\boldsymbol{r}_{j_{\ell}}^{\prime}-\boldsymbol{r}_{\ell}$ the position vector of $M_{j_{\ell}}^{\prime}$ relative to $M_{\ell}$ and by $\Gamma_{\ell, j_{\ell}}$ the covariance matrix of $\boldsymbol{r}_{\ell, j_{\ell}}$ (cf. Appendix A.2),

$\xi_{\ell, j_{\ell}}=\frac{\exp \left(-\frac{1}{2} \boldsymbol{r}_{\ell, j_{\ell}}^{\top} \cdot \Gamma_{\ell, j_{\ell}}^{-1} \cdot \boldsymbol{r}_{\ell, j_{\ell}}\right)}{2 \pi \sqrt{\operatorname{det} \Gamma_{\ell, j_{\ell}}}}$.

If $j_{\ell}=0, M_{\ell}$ is not associated with any source in $K^{\prime}$. Since clustering is neglected,

$P_{: 0}\left(c_{\ell} \mid \bigcap_{k=\ell+1}^{n} c_{k} \cap \bigcap_{k=1}^{n^{\prime}} c_{k}^{\prime} \cap \bigcap_{k=1}^{n} A_{k, j_{k}}\right)=P_{: 0}\left(c_{\ell} \mid A_{\ell, 0}\right)=\xi_{\ell, 0} \mathrm{~d}^{2} \boldsymbol{r}_{\ell}$,

where the last equality defines the spatial probability density $\xi_{\ell, 0}$; for the uninformative prior of a uniform a priori probability distribution of $K$-sources without counterpart, $\xi_{\ell, 0}=1 / S$. From Eqs. (8), (9), and (11), it follows that

$P_{: 0}\left(C \mid \bigcap_{k=1}^{n} A_{k, j_{k}} \cap C^{\prime}\right)=\Xi \prod_{k=1}^{n} \xi_{k, j_{k}}$,

where

$\Xi:=\prod_{k=1}^{n} \mathrm{~d}^{2} \boldsymbol{r}_{k}$

We now compute the second factor in the product of Eq. (6). Knowing the coordinates of $K^{\prime}$-sources alone, without those of any in $K$, does not change the likelihood of the associations $\left(A_{k, j_{k}}\right)$; in other words, $C^{\prime}$ and $\bigcap_{k=1}^{n} A_{k, j_{k}}$ are mutually unconditionally independent (but conditionally dependent on $C$ ). Therefore,

$P_{\text {s:o }}\left(\bigcap_{k=1}^{n} A_{k, j_{k}} \mid C^{\prime}\right)=P_{\text {s:o }}\left(\bigcap_{k=1}^{n} A_{k, j_{k}}\right)$.

Let $q:=\#\left\{k \in \llbracket 1, n \rrbracket \mid j_{k} \neq 0\right\}$, where \#E denotes the number of elements of any set $E$. Since the events $\left(A_{k, j_{k}}\right)_{k \in \llbracket 1, n \rrbracket}$ are independent by assumption $H_{\text {s:o }}$,

$P_{\text {s:o }}\left(\bigcap_{k=1}^{n} A_{k, j_{k}}\right)=\prod_{k=1}^{n} P_{\text {s:o }}\left(A_{k, j_{k}}\right)$.

Using definition (2), and on the hypothesis that all associations $\left(A_{k, \ell}\right)_{\ell \in \llbracket 1, n^{\prime} \rrbracket}$ are a priori equally likely if $k \neq 0$ (Sect. 2.2), we get

$P_{\mathrm{s}: \mathrm{o}}\left(A_{k, j_{k}}\right)=\frac{P_{\mathrm{s}: \mathrm{o}}\left(\bigcup_{\ell \neq 0} A_{k, \ell}\right)}{\# K^{\prime}}=\frac{f}{n^{\prime}} \quad$ for $j_{k} \neq 0$. 
Since $P_{\text {s:o }}\left(A_{k, 0}\right)=1-f$, we have

$P_{\mathrm{s}: \mathrm{o}}\left(\bigcap_{k=1}^{n} A_{k, j_{k}}\right)=\left(\frac{f}{n^{\prime}}\right)^{q}(1-f)^{n-q}$.

Hence, from Eqs. (6), (12) and (17),

$P_{\mathrm{s}: \mathrm{o}}\left(C \mid C^{\prime}\right)=\Xi \sum_{j_{1}=0}^{n^{\prime}} \sum_{j_{2}=0}^{n^{\prime}} \cdots \sum_{j_{n}=0}^{n^{\prime}}\left(\frac{f}{n^{\prime}}\right)^{q}(1-f)^{n-q} \prod_{k=1}^{n} \xi_{k, j_{k}}$.

By the definition of $q$, there are $q$ strictly positive indices $j_{k}$ (as many as the factors " $f / n$ " " in Eq. (18)) and $n-q$ null ones (as many as the factors " $(1-f)$ "). Therefore, with

$\zeta_{k, 0}:=(1-f) \xi_{k, 0} \quad$ and $\quad \zeta_{k, j_{k}}:=\frac{f \xi_{k, j_{k}}}{n^{\prime}} \quad$ for $j_{k} \neq 0$

Eq. (18) reduces to

$P_{\mathrm{s}: \mathrm{o}}\left(C \mid C^{\prime}\right)=\Xi \sum_{j_{1}=0}^{n^{\prime}} \sum_{j_{2}=0}^{n^{\prime}} \cdots \sum_{j_{n}=0}^{n^{\prime}} \prod_{k=1}^{n} \zeta_{k, j_{k}}=\Xi \prod_{k=1}^{n} \sum_{j_{k}=0}^{n^{\prime}} \zeta_{k, j_{k}}$,

where the last equality is derived by induction from the distributivity of multiplication over addition.

\subsubsection{Computation of $P_{\mathrm{s}: 0}\left(A_{i, j} \cap C \mid C^{\prime}\right)$}

The computation of the numerator of Eq. (4) is similar to that of $P_{\text {s:o }}\left(C \mid C^{\prime}\right)$ :

$$
\begin{aligned}
& P_{\text {s:o }}\left(A_{i, j} \cap C \mid C^{\prime}\right) \\
& =P_{\text {s:o }}\left(C \cap A_{i, j} \cap \biguplus_{j_{1}=0}^{n^{\prime}} \cdots \biguplus_{j_{i-1}=0}^{n^{\prime}} \biguplus_{j_{i+1}=0}^{n^{\prime}} \cdots \biguplus_{j_{n}=0}^{n^{\prime}} \prod_{\substack{k=1 \\
k \neq i}}^{n} A_{k, j_{k}} \mid C^{\prime}\right) \\
& =P_{\text {s:o }}\left(C \cap \biguplus_{j_{1}=0}^{n^{\prime}} \cdots \biguplus_{j_{i-1}=0}^{n^{\prime}} \biguplus_{j_{i+1}=0}^{n^{\prime}} \cdots \biguplus_{j_{n}=0}^{n^{\prime}} \bigcap_{k=1}^{n} A_{k, j_{k}} \mid C^{\prime}\right) \\
& =\sum_{j_{1}=0}^{n^{\prime}} \ldots \sum_{j_{i-1}=0}^{n^{\prime}} \sum_{j_{i+1}=0}^{n^{\prime}} \ldots \sum_{j_{n}=0}^{n^{\prime}} P_{\mathrm{s}: \mathrm{o}}\left(C \bigcap_{k=1}^{n} A_{k, j_{k}} \cap C^{\prime}\right) P_{\mathrm{s}: \mathrm{o}}\left(\bigcap_{k=1}^{n} A_{k, j_{k}} \mid C^{\prime}\right),
\end{aligned}
$$

where we put $j_{i}:=j$.

Let $q^{\star}:=\#\left\{k \in \llbracket 1, n \rrbracket \mid j_{k} \neq 0\right\}$ (indices $j_{k}$ are now those of Eq. (21)). As for $P_{\text {s:o }}\left(C \mid C^{\prime}\right)$,

$$
\begin{aligned}
& P_{\mathrm{s}: \mathrm{o}}\left(A_{i, j} \cap C \mid C^{\prime}\right) \\
& =\Xi \sum_{j_{1}=0}^{n^{\prime}} \cdots \sum_{j_{i-1}=0}^{n^{\prime}} \sum_{j_{i+1}=0}^{n^{\prime}} \cdots \sum_{j_{n}=0}^{n^{\prime}}\left(\frac{f}{n^{\prime}}\right)^{q^{\star}}(1-f)^{n-q^{\star}} \prod_{k=1}^{n} \xi_{k, j_{k}} \\
& =\Xi \zeta_{i, j_{i}} \sum_{j_{1}=0}^{n^{\prime}} \cdots \sum_{j_{i-1}=0}^{n^{\prime}} \sum_{j_{i+1}=0}^{n^{\prime}} \cdots \sum_{j_{n}=0}^{n^{\prime}} \prod_{\substack{k=1 \\
k \neq i}}^{n} \zeta_{k, j_{k}}=\Xi \zeta_{i, j} \prod_{\substack{k=1 \\
k \neq i}}^{n} \sum_{j_{k}=0}^{n^{\prime}} \zeta_{k, j_{k}} .
\end{aligned}
$$

\subsubsection{Final results}

Finally, from Eqs. (4), (20), and (22), for $i \neq 0$,

$$
P_{\text {S:o }}\left(A_{i, j} \mid C \cap C^{\prime}\right)=\frac{\zeta_{i, j} \prod_{\substack{k=1 \\ k \neq i}}^{n} \sum_{j_{k}=0}^{n^{\prime}} \zeta_{k, j_{k}}}{\prod_{k=1}^{n} \sum_{j_{k}=0}^{n^{\prime}} \zeta_{k, j_{k}}}=\frac{\zeta_{i, j}}{\sum_{k=0}^{n^{\prime}} \zeta_{i, k}}
$$

$$
= \begin{cases}\frac{f \xi_{i, j}}{(1-f) n^{\prime} \xi_{i, 0}+f \sum_{k=1}^{n^{\prime}} \xi_{i, k}} & \text { for } j \neq 0, \\ \frac{(1-f) n^{\prime} \xi_{i, 0}}{(1-f) n^{\prime} \xi_{i, 0}+f \sum_{k=1}^{n^{\prime}} \xi_{i, k}} & \text { for } j=0 .\end{cases}
$$

As to the probability $P_{\text {s:o }}\left(A_{0, j} \mid C \cap C^{\prime}\right)$ that $M_{j}^{\prime}$ has no counterpart in $K$, it can be computed in this way:

$$
\begin{aligned}
& P_{\mathrm{s}: \mathrm{o}}\left(A_{0, j} \cap C \mid C^{\prime}\right)=P_{\mathrm{s}: \mathrm{o}}\left(C \cap A_{0, j} \cap \biguplus_{j_{1}=0}^{n^{\prime}} \biguplus_{j_{2}=0}^{n^{\prime}} \cdots \biguplus_{j_{n}=0}^{n^{\prime}} \bigcap_{k=1}^{n} A_{k, j_{k}} \mid C^{\prime}\right) \\
& =P_{\text {s:o }}\left(C \cap \biguplus_{\substack{j_{1}=0 \\
j_{1} \neq j}}^{n^{\prime}} \biguplus_{\substack{j_{2}=0 \\
j_{2} \neq j}}^{n^{\prime}} \cdots \biguplus_{\substack{j_{n}=0 \\
j_{n} \neq j}}^{n^{\prime}} \bigcap_{k=1}^{n} A_{k, j_{k}} \mid C^{\prime}\right) \\
& =\sum_{\substack{j_{1}=0 \\
j_{1} \neq j}}^{n^{\prime}} \sum_{j_{2}=0}^{n_{2} \neq j} \cdots \sum_{\substack{j_{n}=0 \\
j_{n} \neq j}}^{n^{\prime}} P_{\mathrm{s}: \mathrm{o}}\left(C \cap \bigcap_{k=1}^{n} A_{k, j_{k}} \mid C^{\prime}\right) \\
& =\Xi \sum_{\substack{j_{1}=0 \\
j_{1} \neq j}}^{n^{\prime}} \sum_{j_{2}=0}^{n_{2} \neq j} \cdots \sum_{\substack{j_{n}=0 \\
j_{n} \neq j}}^{n^{\prime}} \prod_{k=1}^{n} \zeta_{k, j_{k}}=\Xi \prod_{k=1}^{n} \sum_{\substack{j_{k}=0 \\
j_{k} \neq j}}^{n^{\prime}} \zeta_{k, j_{k}}
\end{aligned}
$$

and, using Eqs. (20), (23), and (3),

$$
\begin{gathered}
P_{\mathrm{s}: \mathrm{o}}\left(A_{0, j} \mid C \cap C^{\prime}\right)=\frac{P_{\mathrm{s}: \mathrm{o}}\left(A_{0, j} \cap C \mid C^{\prime}\right)}{P_{\mathrm{s}: \mathrm{o}}\left(C \mid C^{\prime}\right)}=\frac{\Xi \prod_{k=1}^{n} \sum_{\substack{j_{k}=0 \\
j_{k} \neq j}}^{n^{\prime}} \zeta_{k, j_{k}}}{\Xi \prod_{k=1}^{n} \sum_{j_{k}=0}^{n^{\prime}} \zeta_{k, j_{k}}} \\
=\prod_{k=1}^{n} \frac{\sum_{j_{k}=0}^{n^{\prime}} \zeta_{k, j_{k}}-\zeta_{k, j}}{\sum_{j_{k}=0}^{n^{\prime}} \zeta_{k, j_{k}}}=\prod_{k=1}^{n}\left(1-\frac{\zeta_{k, j_{k}}}{\sum_{j_{k}=0}^{n^{\prime}} \zeta_{k, j_{k}}}\right) \\
=\prod_{k=1}^{n}\left(1-P_{\mathrm{s}: \mathrm{o}}\left[A_{k, j} \mid C \cap C^{\prime}\right]\right) \text { for } j \neq 0 .
\end{gathered}
$$

\subsection{Likelihood and estimation of unknown parameters}

\subsubsection{General results}

Various methods have been proposed for estimating the fraction of sources with a counterpart (Kim et al. 2012; Fleuren et al. 2012; McAlpine et al. 2012; Haakonsen \& Rutledge 2009). Pineau et al. (2011), for instance, fit $f$ to the overall distribution of the likelihood ratios. We propose a more convenient and systematic method in this section.

Besides $f$, the probabilities $P\left(A_{i, j} \mid C \cap C^{\prime}\right)$ may depend on other unknowns, such as the parameters $\stackrel{\circ}{\sigma}$ and $\dot{v}$ modeling the positional uncertainties (cf. Appendices A.2.2 and A.2.3). We write here $x_{1}, x_{2}$, etc., for all these parameters, and put $\boldsymbol{x}:=$ $\left(x_{1}, x_{2}, \ldots\right)$. An estimate $\hat{\boldsymbol{x}}$ of $\boldsymbol{x}$ may be obtained by maximizing with respect to $\boldsymbol{x}$ (and with the constraint $\hat{f} \in[0,1]$ ) the overall likelihood

$L:=\frac{P\left(C \cap C^{\prime}\right)}{\left(\prod_{i=1}^{n} \mathrm{~d}^{2} \boldsymbol{r}_{i}\right) \prod_{j=1}^{n^{\prime}} \mathrm{d}^{2} \boldsymbol{r}_{j}^{\prime}}$ 
to observe all the $K$ - and $K^{\prime}$-sources at their effective positions. Unless the result is outside the possible domain for $\boldsymbol{x}$ (i.e., if $L$ reaches its maximum on the boundary of this domain), the maximum likelihood estimator $\hat{\boldsymbol{x}}$ is a solution to

$\left(\frac{\partial \ln L}{\partial x}\right)_{x=\hat{x}}=0$

From now on, all quantities calculated at $\boldsymbol{x}=\hat{\boldsymbol{x}}$ bear a circumflex.

We have

$P\left(C \cap C^{\prime}\right)=P\left(C \mid C^{\prime}\right) P\left(C^{\prime}\right)$,

and, since clustering is neglected,

$P\left(C^{\prime}\right)=\prod_{j=1}^{n^{\prime}} P\left(c_{j}^{\prime}\right)=\prod_{j=1}^{n^{\prime}} \xi_{0, j} \mathrm{~d}^{2} \boldsymbol{r}_{j}^{\prime}$,

where $\xi_{0, j}$ is the spatial probability density defined by $P\left(c_{j}^{\prime}\right)=$ $\xi_{0, j} \mathrm{~d}^{2} \boldsymbol{r}_{j}^{\prime}$; for the uninformative prior of a uniform a priori probability distribution of $K^{\prime}$-sources, $\xi_{0, j}=1 / S$. From Eqs. (27), (29), (30), and (13), we obtain

$L=\frac{P\left(C \mid C^{\prime}\right)}{\Xi} \prod_{j=1}^{n^{\prime}} \xi_{0, j}$

In particular, under assumption $H_{\mathrm{s}: \mathrm{o}}$, Eqs. (31), (20), and (13) give

$L_{\mathrm{s}: \mathrm{o}}=\left(\prod_{i=1}^{n} \sum_{k=0}^{n^{\prime}} \zeta_{i, k}\right) \prod_{j=1}^{n^{\prime}} \xi_{0, j}$.

Therefore, for any parameter $x_{p}$ and because the $\xi_{0, j}$ are independent of $\boldsymbol{x}$,

$$
\begin{aligned}
\frac{\partial \ln L_{\mathrm{s}: \mathrm{o}}}{\partial x_{p}}=\sum_{i=1}^{n} \frac{\partial \ln \sum_{k=0}^{n^{\prime}} \zeta_{i, k}}{\partial x_{p}} & \\
=\sum_{i=1}^{n} \sum_{j=0}^{n^{\prime}} \frac{\partial \zeta_{i, j} / \partial x_{p}}{\sum_{k=0}^{n^{\prime}} \zeta_{i, k}} & =\sum_{i=1}^{n} \sum_{j=0}^{n^{\prime}} \frac{\partial \ln \zeta_{i, j}}{\partial x_{p}} \frac{\zeta_{i, j}}{\sum_{k=0}^{n^{\prime}} \zeta_{i, k}} \\
& =\sum_{i=1}^{n} \sum_{j=0}^{n^{\prime}} \frac{\partial \ln \zeta_{i, j}}{\partial x_{p}} P_{\mathrm{s}: \mathrm{o}}\left(A_{i, j} \mid C \cap C^{\prime}\right)
\end{aligned}
$$

(For reasons highlighted just after Eq. (73), it is convenient to express most results as a function of the probabilities $P\left(A_{i, j} \mid\right.$ $\left.C \cap C^{\prime}\right)$.)

Uncertainties on the unknown parameters may be computed from the covariance matrix $V$ of $\hat{\boldsymbol{x}}$. For large numbers of sources, $V$ is asymptotically given (Kendall \& Stuart 1979) by

$\left(V^{-1}\right)_{p, q}=-\left(\frac{\partial^{2} \ln L}{\partial x_{p} \partial x_{q}}\right)_{\hat{x}=x}$.

\subsubsection{Fraction of sources with a counterpart}

Consider, in particular, the case $x_{p}=f$. We note that

$\frac{\partial \ln \zeta_{i, 0}}{\partial f}=-\frac{1}{1-f} \quad$ and $\quad \frac{\partial \ln \zeta_{i, j}}{\partial f}=\frac{1}{f} \quad$ for $j \neq 0$.
Under the assumption $H_{\mathrm{s}: \mathrm{o}}$ or $H_{\mathrm{o}: \mathrm{o}}$ (but not under $H_{\mathrm{o}: \mathrm{s}}$ ),

$\sum_{j=0}^{n^{\prime}} P_{: \mathrm{o}}\left(A_{i, j} \mid C \cap C^{\prime}\right)=P_{: \mathrm{o}}\left(\biguplus_{j=0}^{n^{\prime}} A_{i, j} \mid C \cap C^{\prime}\right)=1$,

so, using Eq. (35),

$$
\begin{aligned}
& \sum_{j=0}^{n^{\prime}} \frac{\partial \ln \zeta_{i, j}}{\partial f} P_{: \mathrm{o}}\left(A_{i, j} \mid C \cap C^{\prime}\right) \\
& =-\frac{P_{: \mathrm{o}}\left(A_{i, 0} \mid C \cap C^{\prime}\right)}{1-f}+\sum_{j=1}^{n^{\prime}} \frac{P_{: \mathrm{o}}\left(A_{i, j} \mid C \cap C^{\prime}\right)}{f} \\
& =-\frac{P_{: \mathrm{o}}\left(A_{i, 0} \mid C \cap C^{\prime}\right)}{1-f}+\frac{1-P_{: \mathrm{o}}\left(A_{i, 0} \mid C \cap C^{\prime}\right)}{f} \\
& =\frac{(1-f)-P_{: 0}\left(A_{i, 0} \mid C \cap C^{\prime}\right)}{f(1-f)} .
\end{aligned}
$$

Summing Eq. (37) on $i$, we obtain from Eq. (33) that

$\frac{\partial \ln L_{\mathrm{s}: \mathrm{o}}}{\partial f}=\frac{n(1-f)-\sum_{i=1}^{n} P_{\mathrm{s}: \mathrm{o}}\left(A_{i, 0} \mid C \cap C^{\prime}\right)}{f(1-f)}$.

Consequently, the maximum likelihood estimator of the fraction $f$ of $K$-sources with a counterpart in $K^{\prime}$ is

$$
\begin{aligned}
\hat{f}_{\mathrm{s}: \mathrm{o}} & =1-\frac{1}{n} \sum_{i=1}^{n} \hat{P}_{\mathrm{s}: \mathrm{o}}\left(A_{i, 0} \mid C \cap C^{\prime}\right) \\
& =\frac{1}{n} \sum_{i=1}^{n} \sum_{j=1}^{n^{\prime}} \hat{P}_{\mathrm{s}: \mathrm{o}}\left(A_{i, j} \mid C \cap C^{\prime}\right) .
\end{aligned}
$$

After some tedious calculations, it can be shown that

$$
\frac{\partial^{2} \ln L_{\mathrm{s}: \mathrm{o}}}{\partial f^{2}}=-\frac{\sum_{i=1}^{n}\left([1-f]-P_{\mathrm{s}: \mathrm{o}}\left[A_{i, 0} \mid C \cap C^{\prime}\right]\right)^{2}}{f^{2}(1-f)^{2}}<0
$$

for all $f$, so $\partial \ln L_{\mathrm{s}: \mathrm{o}} / \partial f$ has at most one zero in $[0,1]: \hat{f}_{\mathrm{s}: \mathrm{o}}$ is unique.

Since $\hat{f}_{\mathrm{s}: o}$ appears on the two sides of Eq. (39) (remember that $\hat{P}_{\text {s:o }}$ is the value of $P_{\text {s:o }}$ at $f=\hat{f}_{\text {s:o }}$ ), we may try to determine it through an iterative back and forth computation between the lefthand and the righthand sides of this equation. (A similar idea was also proposed by Benn 1983.) We prove in Sect. 5.3 that this procedure converges for any starting value $f \in] 0,1[$.

An estimate $\hat{f}_{\text {s:o }}^{\prime}$ of the fraction $f^{\prime}$ of $K^{\prime}$-sources with a counterpart is given by

$\hat{f}_{\mathrm{s}: \mathrm{o}}^{\prime}=1-\frac{1}{n^{\prime}} \sum_{j=1}^{n^{\prime}} \hat{P}_{\mathrm{s}: \mathrm{o}}\left(A_{0, j} \mid C \cap C^{\prime}\right)$.

It can be checked from Eqs. (40), (42), and (26) that, as expected if assumption $H_{\text {s:o }}$ is valid (cf. Sect. 2.2), $\hat{f}_{\text {s:o }} n \geqslant \hat{f}_{\text {s:o }}^{\prime} n^{\prime}$. (Just notice that, for any numbers $y_{i} \in[0,1], \prod_{i=1}^{n}\left(1-y_{i}\right) \geqslant 1-\sum_{i=1}^{n} y_{i}$, which is obvious by induction; apply this to $y_{i}=\hat{P}_{\mathrm{s}: \mathrm{o}}\left(A_{i, j}\right.$ । $C \cap C^{\prime}$ ) and then sum on $j$.) 


\subsection{Probability of association: local computation}

Under assumption $H_{\text {s:o }}$, a purely local computation (subscript "loc" hereafter) of the probabilities of association is also possible. Consider a region $U_{i}$ of area $S_{i}$ containing the position of $M_{i}$, and such that we can safely hypothesize that the counterpart in $K^{\prime}$ of $M_{i}$, if any, is inside. We assume that the local surface density $\rho_{i}^{\prime}$ of $K^{\prime}$-sources unrelated to $M_{i}$ is uniform on $U_{i}$. To avoid biasing the estimate if $M_{i}$ has a counterpart, $\rho_{i}^{\prime}$ may be evaluated from the number of $K^{\prime}$-sources in a region surrounding $U_{i}$, but not overlapping it (an annulus around a disk $U_{i}$ centered on $M_{i}$, for instance).

Besides the $A_{i, j}$, we consider the following events:

- $N_{i}^{\prime}: U_{i}$ contains $n_{i}^{\prime}$ sources;

- $C_{i}^{\prime}:=\bigcap_{j \in J_{i}} c_{j}^{\prime}$, where $J_{i}:=\left\{j \mid M_{j}^{\prime} \in U_{i}\right\}$.

We want to compute the probability that a source $M_{j}^{\prime}$ in $U_{i}$ is the counterpart of $M_{i}$, given the positions relative to $M_{i}$ of all its possible counterparts $\left(M_{k}^{\prime}\right)_{k \in J_{i}}$, i.e. $P_{\text {loc }}\left(A_{i, j} \mid C_{i}^{\prime} \cap N_{i}^{\prime}\right)$. Using Eq. (3) with $\omega_{1}=A_{i, j}, \omega_{2}=C_{i}^{\prime}$, and $\omega_{3}=N_{i}^{\prime}$ in the first equality below, and then with $\omega_{1}=C_{i}^{\prime}, \omega_{2}=A_{i, k}$, and $\omega_{3}$ unchanged in the last one, we obtain

$$
\begin{gathered}
P_{\mathrm{loc}}\left(A_{i, j} \mid C_{i}^{\prime} \cap N_{i}^{\prime}\right)=\frac{P_{\mathrm{loc}}\left(A_{i, j} \cap C_{i}^{\prime} \mid N_{i}^{\prime}\right)}{P_{\mathrm{loc}}\left(C_{i}^{\prime} \mid N_{i}^{\prime}\right)} \\
=\frac{P_{\mathrm{loc}}\left(C_{i}^{\prime} \cap A_{i, j} \mid N_{i}^{\prime}\right)}{P_{\mathrm{loc}}\left(C_{i}^{\prime} \cap \biguplus_{k \in J_{i} \cup\{0\}} A_{i, k} \mid N_{i}^{\prime}\right)}=\frac{P_{\mathrm{loc}}\left(C_{i}^{\prime} \cap A_{i, j} \mid N_{i}^{\prime}\right)}{\sum_{k \in J_{i} \cup\{0\}} P_{\mathrm{loc}}\left(C_{i}^{\prime} \cap A_{i, k} \mid N_{i}^{\prime}\right)} \\
=\frac{P_{\mathrm{loc}}\left(C_{i}^{\prime} \mid A_{i, j} \cap N_{i}^{\prime}\right) P_{\mathrm{loc}}\left(A_{i, j} \mid N_{i}^{\prime}\right)}{\sum_{k \in J_{i} \cup\{0\}} P_{\mathrm{loc}}\left(C_{i}^{\prime} \mid A_{i, k} \cap N_{i}^{\prime}\right) P_{\mathrm{loc}}\left(A_{i, k} \mid N_{i}^{\prime}\right)}
\end{gathered}
$$

Now,

$$
\begin{aligned}
P_{\mathrm{loc}}\left(A_{i, 0} \mid N_{i}^{\prime}\right)=\frac{P_{\mathrm{loc}}\left(N_{i}^{\prime} \cap A_{i, 0}\right)}{P_{\mathrm{loc}}\left(N_{i}^{\prime}\right)} \\
=\frac{P_{\mathrm{loc}}\left(N_{i}^{\prime} \mid A_{i, 0}\right) P_{\mathrm{loc}}\left(A_{i, 0}\right)}{P_{\mathrm{loc}}\left(N_{i}^{\prime} \mid A_{i, 0}\right) P_{\mathrm{loc}}\left(A_{i, 0}\right)+P_{\mathrm{loc}}\left(N_{i}^{\prime} \mid \overline{A_{i, 0}}\right) P_{\mathrm{loc}}\left(\overline{A_{i, 0}}\right)}
\end{aligned}
$$

and

$$
P_{\mathrm{loc}}\left(A_{i, j} \mid N_{i}^{\prime}\right)=\frac{P_{\mathrm{loc}}\left(\overline{A_{i, 0}} \mid N_{i}^{\prime}\right)}{n_{i}^{\prime}}=\frac{1-P_{\mathrm{loc}}\left(A_{i, 0} \mid N_{i}^{\prime}\right)}{n_{i}^{\prime}} \quad \text { for } j \neq 0 .
$$

(The probability $P_{\text {loc }}\left(A_{i, j}\right)$ itself could not have been computed as $P_{\text {loc }}\left(\overline{A_{i, 0}}\right) / n_{i}^{\prime}$ because $n_{i}^{\prime}$ would be undefined, which is why event $N_{i}^{\prime}$ was introduced.) If clustering is negligible, the number of $K^{\prime}$-sources randomly distributed with a mean surface density $\rho_{i}^{\prime}$ in an area $S_{i}$ follows a Poissonian distribution, so

$$
P_{\mathrm{loc}}\left(N_{i}^{\prime} \mid \overline{A_{i, 0}}\right)=\frac{\left(\rho_{i}^{\prime} S_{i}\right)^{n_{i}^{\prime}-1} \exp \left(-\rho_{i}^{\prime} S_{i}\right)}{\left(n_{i}^{\prime}-1\right) !}
$$

(one counterpart and $n_{i}^{\prime}-1$ sources by chance in $S_{i}$ ) and

$$
P_{\mathrm{loc}}\left(N_{i}^{\prime} \mid A_{i, 0}\right)=\frac{\left(\rho_{i}^{\prime} S_{i}\right)^{n_{i}^{\prime}} \exp \left(-\rho_{i}^{\prime} S_{i}\right)}{n_{i}^{\prime} !}
$$

(no counterpart and $n_{i}^{\prime}$ sources by chance in $S_{i}$ ). Thus, from Eqs. (45)-(47), and (2),

$P_{\mathrm{loc}}\left(A_{i, j} \mid N_{i}^{\prime}\right)= \begin{cases}\frac{f}{n_{i}^{\prime} f+(1-f) \rho_{i}^{\prime} S_{i}} & \text { for } j \neq 0, \\ \frac{(1-f) \rho_{i}^{\prime} S_{i}}{n_{i}^{\prime} f+(1-f) \rho_{i}^{\prime} S_{i}} & \text { for } j=0 .\end{cases}$
We have

$$
\begin{aligned}
& P_{\mathrm{loc}}\left(C_{i}^{\prime} \mid A_{i, 0} \cap N_{i}^{\prime}\right)=\prod_{k \in J_{i}} \frac{\mathrm{d}^{2} \boldsymbol{r}_{k}^{\prime}}{S_{i}} \quad \text { and } \\
& P_{\mathrm{loc}}\left(C_{i}^{\prime} \mid A_{i, j} \cap N_{i}^{\prime}\right)=\xi_{i, j} \mathrm{~d}^{2} \boldsymbol{r}_{j}^{\prime} \prod_{\substack{k \in J_{i} \\
k \neq j}} \frac{\mathrm{d}^{2} \boldsymbol{r}_{k}^{\prime}}{S_{i}} \text { for } j \neq 0
\end{aligned}
$$

(rigorously, $\xi_{i, j}$ should be replaced by $\xi_{i, j} / P_{\text {loc }}\left(M_{j}^{\prime} \in U_{i} \mid A_{i, j}\right)$, but $P_{\text {loc }}\left(M_{j}^{\prime} \notin U_{i} \mid A_{i, j}\right)$ is negligible by definition of $\left.U_{i}\right)$, so, using Eqs. (43), (48), and (49), we obtain

$$
P_{\mathrm{loc}}\left(A_{i, j} \mid C_{i}^{\prime} \cap N_{i}^{\prime}\right)= \begin{cases}\frac{f \lambda_{i, j}}{(1-f)+f \sum_{k \in J_{i}} \lambda_{i, k}} & \text { for } j \neq 0, \\ \frac{(1-f)}{(1-f)+f \sum_{k \in J_{i}} \lambda_{i, k}} & \text { for } j=0,\end{cases}
$$

where $\lambda_{i, k}:=\xi_{i, k} / \rho_{i}^{\prime}$ is the likelihood ratio (cf. Eq. (1)). Mutatis mutandis, we obtain the same result as Eq. (14) of Pineau et al. (2011) and the aforementioned authors. When the computation is extended from $U_{i}$ to the whole surface covered by $K^{\prime}, \rho_{i}^{\prime}$ is replaced by $n^{\prime} / S$ in Eq. (50), $\sum_{k \in J_{i}}$ by $\sum_{k=1}^{n^{\prime}}$, and we recover Eq. (24) since $\xi_{i, 0}=1 / S$ for a uniform distribution.

The index $j_{\mathrm{MLC}}(i)$ of the most likely counterpart $M_{j_{\mathrm{MLC}}(i)}^{\prime}$ of $M_{i}$ is the value of $j \neq 0$ maximizing $\lambda_{i, j}$. Very often, $\lambda_{i, j_{\mathrm{MLC}}(i)}^{j_{\mathrm{MLC}}(i)} \gg$ $\sum_{k \in J_{i} ; k \neq j_{\mathrm{MLC}}(i)} \lambda_{i, k}$, so

$P_{\mathrm{s}: 0}\left(A_{i, j_{\mathrm{MLC}}(i)} \mid C_{i}^{\prime} \cap N_{i}^{\prime}\right) \approx \frac{f \lambda_{i, j_{\mathrm{MLC}}(i)}}{(1-f)+f \lambda_{i, j_{\mathrm{MLC}}(i)}}$.

As a "poor man's" recipe, if the value of $f$ is unknown and not too close to either 0 or 1 , an association may be considered as true if $\lambda_{i, j_{\mathrm{MLC}}(i)} \gg 1$ and as false if $\lambda_{i, j_{\mathrm{MLC}}(i)} \ll 1$. Where to set the boundary between true associations and false ones is somewhat arbitrary (Wolstencroft et al. 1986). For a large sample, however, $f$ can be estimated from the distribution of the positions of all sources, as shown in Sect. 3.2.

\section{One-to-one associations}

Under $H_{\text {s:o }}$ (Sect. 3), a given $M_{j}^{\prime}$ can be associated with several $M_{i}$ : there is no symmetry between $K$ and $K^{\prime}$ under this assumption and, while $\sum_{j=0}^{n^{\prime}} P_{\mathrm{s}: \mathrm{o}}\left(A_{i, j} \mid C \cap C^{\prime}\right)=1$ for all $M_{i}$, $\sum_{i=1}^{n} P_{\text {s:o }}\left(A_{i, j} \mid C \cap C^{\prime}\right)$ could be strictly larger than 1 for some sources $M_{j}^{\prime}$. We assume here that the much more constraining assumption $H_{\mathrm{o}: o}$ holds. As far as we know and despite some attempt by Rutledge et al. (2000), this problem has not been solved previously (see also Bartlett \& Egret 1998 for a simple statement of the question).

Since a $K^{\prime}$-potential counterpart $M_{j}^{\prime}$ of $M_{i}$ within some neighborhood $U_{i}$ of $M_{i}$ might in fact be the true counterpart of another source $M_{k}$ outside of $U_{i}$, there is no obvious way to adapt the exact local several-to-one computation of Sect. 3.3 to the case of the one-to-one assumption. We therefore have to consider all the $K$ - and $K^{\prime}$-sources, as in Sect. 3.1.

Under assumption $H_{\mathrm{o}: 0}$, catalogs $K$ and $K^{\prime}$ play symmetrical roles; in particular,

$P_{\mathrm{o:o}}\left(A_{i, j}\right)=\frac{f}{n^{\prime}}=\frac{f^{\prime}}{n} \quad$ if $i \neq 0$ and $j \neq 0$.

For practical reasons (cf. Eq. (61)), we nonetheless name $K$ the catalog with the fewer objects and $K^{\prime}$ the other one, so $n \leqslant n^{\prime}$ in the following. 


\subsection{Probability of association}

\subsubsection{Computation of $P_{\mathrm{o}: 0}\left(C \mid C^{\prime}\right)$}

The denominator of Eq. (4) is

$P_{\text {o:o }}\left(C \mid C^{\prime}\right)=P_{\text {o:o }}\left(C \cap \biguplus_{j_{1}=0}^{n^{\prime}} \biguplus_{j_{2}=0}^{n^{\prime}} \cdots \biguplus_{j_{n}=0}^{n^{\prime}} \bigcap_{k=1}^{n} A_{k, j_{k}} \mid C^{\prime}\right)$

(same reasons as for Eq. (6)). Because $A_{k, m} \cap A_{\ell, m}=\varnothing$ if $k \neq \ell$ and $m \neq 0$ by assumption $H_{\mathrm{o}: 0}$, this reduces to

$P_{\mathrm{o}: \mathrm{o}}\left(C \mid C^{\prime}\right)=P_{\mathrm{o}: \mathrm{o}}\left(C \cap \biguplus_{\substack{j_{1}=0 \\ j_{1} \notin X_{0}}}^{n^{\prime}} \biguplus_{j_{2}=0}^{j_{2} \notin X_{1}} \underset{\substack{n_{n}=0 \\ j_{n} \notin X_{n-1}}}{n_{k=1}^{n^{\prime}}} \bigcap_{k=1}^{n} A_{k, j_{k}} \mid C^{\prime}\right)$,

where, to ensure that each $K^{\prime}$-source is associated with at most one of $K$, the sets $X_{k}$ of excluded counterparts are defined iteratively by

$X_{0}:=\varnothing \quad$ and $\quad X_{k}:=\left(X_{k-1} \cup\left\{j_{k}\right\}\right) \backslash\{0\} \quad$ for all $k \in \llbracket 1, n \rrbracket$.

As a result,

$$
\begin{aligned}
& P_{\mathrm{o}: \mathrm{o}}\left(C \mid C^{\prime}\right)=\sum_{\substack{j_{1}=0 \\
j_{1} \notin X_{0}}}^{n_{0}} \sum_{j_{2}=0}^{j_{2} \notin X_{1}} \cdots \sum_{\substack{j_{n}=0 \\
j_{n} \notin X_{n-1}}}^{n^{\prime}} P_{\mathrm{o:o}}\left(C \cap \bigcap_{k=1}^{n} A_{k, j_{k}} \mid C^{\prime}\right) \\
& =\sum_{\substack{j_{1}=0 \\
j_{1} \notin X_{0}}}^{n^{\prime}} \sum_{\substack{j_{2}=0 \\
j_{2} \notin X_{1}}}^{n^{\prime}} \cdots \sum_{\substack{j_{n}=0 \\
j_{n} \notin X_{n-1}}}^{n^{\prime}} P_{\mathrm{o}: \mathrm{o}}\left(C \mid \bigcap_{k=1}^{n} A_{k, j_{k}} \cap C^{\prime}\right) P_{\mathrm{o}: \mathrm{o}}\left(\bigcap_{k=1}^{n} A_{k, j_{k}} \mid C^{\prime}\right) .
\end{aligned}
$$

The first factor in the product of Eq. (56) is still given by Eq. (12), so we just have to compute the second factor,

$$
P_{\text {o:o }}\left(\bigcap_{k=1}^{n} A_{k, j_{k}} \mid C^{\prime}\right)=P_{\text {o:o }}\left(\bigcap_{k=1}^{n} A_{k, j_{k}}\right) \text {. }
$$

Let $q:=\# X_{n}$ and $Q$ be a random variable describing the number of associations between $K$ and $K^{\prime}$ :

$$
\begin{aligned}
P_{\mathrm{o}: \mathrm{o}}\left(\bigcap_{k=1}^{n} A_{k, j_{k}}\right)=P_{\mathrm{o}: \mathrm{o}} & \left(\bigcap_{k=1}^{n} A_{k, j_{k}} \mid Q=q\right) P_{\mathrm{o}: \mathrm{o}}(Q=q) \\
& +P_{\mathrm{o}: \mathrm{o}}\left(\bigcap_{k=1}^{n} A_{k, j_{k}} \mid Q \neq q\right) P_{\mathrm{o}: \mathrm{o}}(Q \neq q) .
\end{aligned}
$$

Since $P_{\text {o:o }}\left(\bigcap_{k=1}^{n} A_{k, j_{k}} \mid Q \neq q\right)=0$ by definition of $q$, we only have to compute $P_{\mathrm{o}: \mathrm{o}}\left(\bigcap_{k=1}^{n} A_{k, j_{k}} \mid Q=q\right)$ and $P_{\mathrm{o}: \mathrm{o}}(Q=q)$.

There are $n ! /(q$ ! $[n-q] !)$ choices of $q$ elements among $n$ in $K$, and $n^{\prime} ! /\left(q\right.$ ! $\left[n^{\prime}-q\right]$ !) choices of $q$ elements among $n^{\prime}$ in $K^{\prime}$. The number of permutations of $q$ elements is $q$ !, so the total number of one-to-one associations of $q$ elements from $K$ to $q$ elements of $K^{\prime}$ is

$q ! \frac{n !}{q !(n-q) !} \frac{n^{\prime} !}{q !\left(n^{\prime}-q\right) !}$

The inverse of this number is

$P_{\mathrm{o}: \mathrm{o}}\left(\bigcap_{k=1}^{n} A_{k, j_{k}} \mid Q=q\right)=\frac{q !(n-q) !\left(n^{\prime}-q\right) !}{n ! n^{\prime} !}$.
With our definition of $K$ and $K^{\prime}, n \leqslant n^{\prime}$, so all the elements of $K$ may have a counterpart in $K^{\prime}$ jointly. Therefore, $P_{\mathrm{o}: \mathrm{o}}(Q=q)$ is given by the binomial law:

$P_{\mathrm{o}: \mathrm{o}}(Q=q)=\frac{n !}{q !(n-q) !} f^{q}(1-f)^{n-q}$.

From Eqs. (56), (12), (60), and (61), we obtain

$P_{\mathrm{o:o}}\left(C \mid C^{\prime}\right)$

$$
\begin{aligned}
& =\Xi \sum_{\substack{j_{1}=0 \\
j_{1} \notin X_{0}}}^{n^{\prime}} \sum_{\substack{j_{2}=0 \\
j_{2} \notin X_{1}}}^{n^{\prime}} \cdots \sum_{\substack{j_{n}=0 \\
j_{n} \notin X_{n-1}}}^{n^{\prime}} \frac{\left(n^{\prime}-q\right) !}{n^{\prime} !} f^{q}(1-f)^{n-q} \prod_{k=1}^{n} \xi_{k, j_{k}} \\
& =\Xi \sum_{\substack{j_{1}=0 \\
j_{1} \notin X_{0}}}^{n^{\prime}} \sum_{\substack{j_{2}=0 \\
j_{2} \notin X_{1}}}^{n^{\prime}} \cdots \sum_{\substack{j_{n}=0 \\
j_{n} \notin X_{n-1}}}^{n^{\prime}}\left(\prod_{\ell=1}^{q} \frac{f}{n^{\prime}-\ell+1}\right)\left(\prod_{\ell=1}^{n-q}[1-f]\right) \prod_{k=1}^{n} \xi_{k, j_{k}} .
\end{aligned}
$$

There are $q$ factors " $f /\left(n^{\prime}-\ell+1\right)$ " in the above equation, one for each index $j_{k} \neq 0$. There are also $n-q$ factors " $(1-f)$ ",

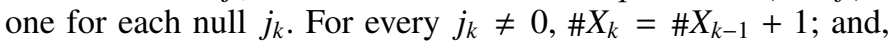
since $q=\# X_{n}$, a different $j_{k}$ corresponds to each $\ell \in \llbracket 1, q \rrbracket$, so $\ell=\# X_{k}$. With

$\eta_{k, 0}:=\zeta_{k, 0} \quad$ and $\quad \eta_{k, j_{k}}:=\frac{f \xi_{k, j_{k}}}{n^{\prime}-\# X_{k-1}} \quad$ for $j_{k} \neq 0$,

Eq. (63) therefore simplifies to

$P_{\text {o:o }}\left(C \mid C^{\prime}\right)=\Xi \sum_{\substack{j_{1}=0 \\ j_{1} \notin X_{0}}}^{n^{\prime}} \sum_{\substack{j_{2}=0 \\ j_{2} \notin X_{1}}}^{n^{\prime}} \cdots \sum_{\substack{j_{n}=0 \\ j_{n} \notin X_{n-1}}}^{n^{\prime}} \prod_{k=1}^{n} \eta_{k, j_{k}}$.

\subsubsection{Computation of $P_{\mathrm{o}: 0}\left(A_{i, j} \cap C \mid C^{\prime}\right)$}

The denominator of Eq. (4) is computed in the same way as $P_{\mathrm{o:o}}\left(C \mid C^{\prime}\right)$ :

$$
\begin{aligned}
& P_{\mathrm{o}: \mathrm{o}}\left(A_{i, j} \cap C \mid C^{\prime}\right) \\
& =P_{\mathrm{o}: \mathrm{o}}\left(C \cap A_{i, j} \cap \biguplus_{\substack{j_{1}=0 \\
j_{1} \notin X_{0}^{\star}}}^{n^{\prime}} \cdots \biguplus_{\substack{j_{i-1}=0 \\
j_{i-1} \notin X_{i-2}^{\star}}}^{n^{\prime}} \biguplus_{\substack{j_{i+1}=0 \\
j_{i+1} \notin X_{i}^{\star}}}^{n^{\prime}} \cdots \biguplus_{\substack{j_{n}=0 \\
j_{n} \notin X_{n-1}^{\star}}}^{n^{\prime}} \bigcap_{\substack{k=1 \\
k \neq i}}^{n} A_{k, j_{k}} \mid C^{\prime}\right) \\
& =P_{\mathrm{o}: \mathrm{o}}\left(C \cap \biguplus_{\substack{j_{1}=0 \\
j_{1} \notin X_{0}^{\star}}}^{n^{\prime}} \cdots \biguplus_{\substack{j_{i-1}=0 \\
j_{i-1} \notin X_{i-2}^{\star}}}^{n^{\prime}} \biguplus_{j_{i+1}=0}^{j_{i+1} \notin X_{i}^{\star}} \underset{\substack{j_{n} \\
j_{n} \notin X_{n-1}^{\star}}}{n_{k=1}^{n^{\prime}}} \bigcap_{k=j_{k}}^{n} A_{k} \mid C^{\prime}\right) \text {, }
\end{aligned}
$$

where

$$
\begin{aligned}
X_{0}^{\star}:=\{j\} \backslash\{0\}, \quad j_{i}:=j \quad \text { and } \\
X_{k}^{\star}:=\left(X_{k-1}^{\star} \cup\left\{j_{k}\right\}\right) \backslash\{0\} \quad \text { for all } k \in \llbracket 1, n \rrbracket,
\end{aligned}
$$

so

$$
\begin{aligned}
& P_{\text {o:o }}\left(A_{i, j} \cap C \mid C^{\prime}\right) \\
& =\sum_{\substack{j_{1}=0 \\
j_{1} \notin X_{0}^{\star}}}^{n^{\prime}} \cdots \sum_{\substack{j_{i-1}=0 \\
j_{i-1} \notin X_{i-2}^{\star}}}^{n^{\prime}} \sum_{\substack{j_{i+1}=0 \\
j_{i+1} \notin X_{i}^{\star}}}^{n^{\prime}} \cdots \sum_{\substack{j_{n}=0 \\
j_{n} \notin X_{n-1}^{\star}}}^{n^{\prime}} P_{\text {o:o }}\left(C \mid \bigcap_{k=1}^{n} A_{k, j_{k}} \cap C^{\prime}\right) \\
& \times P_{\mathrm{o}: \mathrm{o}}\left(\bigcap_{k=1}^{n} A_{k, j_{k}} \mid C^{\prime}\right) .
\end{aligned}
$$


Let $q^{\star}:=\# X_{n}^{\star}$. As for $P_{\mathrm{o}: \mathrm{o}}\left(C \mid C^{\prime}\right)$,

$$
\begin{aligned}
& P_{\mathrm{o}: \mathrm{o}}\left(A_{i, j} \cap C \mid C^{\prime}\right) \\
& =\Xi \sum_{\substack{j_{1}=0 \\
j_{1} \notin X_{0}^{\star}}}^{n^{\prime}} \cdots \sum_{\substack{j_{i-1}=0 \\
j_{i-1} \notin X_{i-2}^{\star}}}^{n^{\prime}} \sum_{\substack{j_{i+1}=0 \\
j_{i+1} \notin X_{i}^{\star}}}^{n^{\prime}} \cdots \sum_{\substack{j_{n}=0 \\
j_{n} \notin X^{\star}}}^{n^{\prime}} \frac{\left(n^{\prime}-q^{\star}\right) !}{n^{\prime} !} f^{q^{\star}}(1-f)^{n-q^{\star}} \prod_{k=1}^{n} \xi_{k, j_{k}} \\
& =\Xi \zeta_{i, j} \sum_{\substack{j_{1}=0 \\
j_{1} \notin X_{0}^{\star}}}^{n^{\prime}} \cdots \sum_{\substack{j_{i-1}=0 \\
j_{i-1} \notin X_{i-2}^{\star}}}^{n^{\prime}} \sum_{\substack{j_{i+1}=0 \\
j_{i+1} \notin X_{i}^{\star}}}^{n^{\prime}} \cdots \sum_{\substack{j_{n}=0 \\
j_{n} \notin X_{n-1}^{\star}}}^{n^{\prime}} \prod_{\substack{k=1 \\
k \neq i}}^{n} \eta_{k, j_{k}}^{\star},
\end{aligned}
$$

where

$$
\eta_{k, 0}^{\star}:=\zeta_{k, 0} \quad \text { and } \quad \eta_{k, j_{k}}^{\star}:=\frac{f \xi_{k, j_{k}}}{n^{\prime}-\# X_{k-1}^{\star}} \quad \text { for } j_{k} \neq 0 .
$$

\subsubsection{Final results}

Finally, from Eqs. (4), (65), and (69), for $i \neq 0$,

$$
\begin{aligned}
& P_{\mathrm{o}: \mathrm{o}}\left(A_{i, j} \mid C \cap C^{\prime}\right) \\
& =\frac{\zeta_{i, j} \sum_{\substack{j_{1}=0 \\
n^{\prime}}} \cdots \sum_{\substack{j_{i-1}=0 \\
j_{1} \notin X_{0}^{\star}}}^{j_{i-1}^{\prime} \notin X_{i-2}^{\star}} \sum_{\substack{j_{i+1}=0 \\
j_{i+1} \notin X_{i}^{\star}}} \cdots \sum_{\substack{j_{n}=0 \\
j_{n} \notin X_{n-1}^{\star}}}^{n^{\prime}} \prod_{\substack{k=1 \\
k \neq i}}^{n} \eta_{k, j_{k}}^{\star}}{\sum_{\substack{j_{1}=0 \\
j_{1} \notin X_{0}}}^{\sum_{\substack{j_{2}=0 \\
j_{2} \notin X_{1}}}^{n^{\prime}} \cdots \sum_{\substack{j_{n}=0 \\
j_{n} \notin X_{n-1}}}^{n^{\prime}} \prod_{k=1}^{n} \eta_{k, j_{k}}}} .
\end{aligned}
$$

The probability that a source $M_{j}^{\prime}$ has no counterpart in $K$ is simply given by

$P_{\mathrm{o:o}}\left(A_{0, j} \mid C \cap C^{\prime}\right)=1-\sum_{k=1}^{n} P_{\mathrm{o:o}}\left(A_{k, j} \mid C \cap C^{\prime}\right)$.

\subsection{Likelihood and estimation of unknown parameters}

As in Sect. 3.2, an estimate $\hat{\boldsymbol{x}}_{\mathrm{o}: \mathrm{o}}$ of the set $\boldsymbol{x}$ of unknown parameters may be obtained by solving Eq. (28). Under assumption $H_{\text {o:o }}$, we obtain from Eqs. (65), (31), and (13) that

$L_{\mathrm{o}: \mathrm{o}}=\left(\sum_{\substack{j_{1}=0 \\ j_{1} \notin X_{0}}}^{n^{\prime}} \sum_{\substack{j_{2}=0 \\ j_{2} \notin X_{1}}}^{n^{\prime}} \cdots \sum_{\substack{j_{n}=0 \\ j_{n} \notin X_{n-1}}}^{n^{\prime}} \prod_{k=1}^{n} \eta_{k, j_{k}}\right) \prod_{k=1}^{n^{\prime}} \xi_{0, k}$.

Because the number of terms in Eq. (73) grows exponentially with $n$ and $n^{\prime}$, this equation seems useless. In fact, the prior computation of $L_{\mathrm{o}: \mathrm{o}}$ is not necessary if the probabilities $P_{\mathrm{o}: \mathrm{o}}\left(A_{i, j} \mid\right.$ $C \cap C^{\prime}$ ) are calculable (we see how to evaluate these in Sect. 5.4). Indeed, for any parameter $x_{p}$, we get the same result (Eq. (33)) as under assumption $H_{\text {s:o }}$. First, we note that, since the $\xi_{0, j}$ are independent of $\boldsymbol{x}$, we obtain from Eq. (31) that

$\frac{\partial \ln L}{\partial x_{p}}=\frac{1}{P\left(C \mid C^{\prime}\right)} \frac{\partial P\left(C \mid C^{\prime}\right)}{\partial x_{p}}$.

Now, for any set $\Upsilon$ of indices and any product of strictly positive functions $h_{k}$ of some variable $y$,

$$
\frac{\partial \prod_{k \in \Upsilon} h_{k}}{\partial y}=\sum_{\ell \in \Upsilon} \frac{\partial h_{\ell}}{\partial y} \prod_{\substack{k \in \Upsilon \\ k \neq \ell}} h_{k}=\sum_{\ell \in \Upsilon} \frac{\partial \ln h_{\ell}}{\partial y} \prod_{k \in \Upsilon} h_{k} .
$$

With $h_{k}=\eta_{k, j_{k}}, y=x_{p}$ and $\Upsilon=\llbracket 1, n \rrbracket$, we therefore obtain from Eq. (65) that

$$
\frac{\partial P_{\text {o:o }}\left(C \mid C^{\prime}\right)}{\partial x_{p}}=\Xi \sum_{\substack{j_{1}=0 \\ j_{1} \notin X_{0}}}^{n^{\prime}} \sum_{\substack{j_{2}=0 \\ j_{2} \notin X_{1}}}^{n^{\prime}} \cdots \sum_{\substack{j_{n}=0 \\ j_{n} \notin X_{n-1}}}^{n^{\prime}} \sum_{i=1}^{n} \frac{\partial \ln \eta_{i, j_{i}}}{\partial x_{p}} \prod_{k=1}^{n} \eta_{k, j_{k}} .
$$

The expression of $P_{\mathrm{o}: \mathrm{o}}\left(A_{i, j} \cap C \mid C^{\prime}\right)$ (Eq. (69)) may also be written

$$
P_{\mathrm{o:o}}\left(A_{i, j} \cap C \mid C^{\prime}\right)=\Xi \sum_{\substack{j_{1}=0 \\ j_{1} \notin X_{0}}}^{n^{\prime}} \sum_{j_{2}=0}^{n_{2} \notin X_{1}} \ldots \sum_{\substack{j_{n}=0 \\ j_{n} \notin X_{n-1}}}^{n^{\prime}} \chi\left(j_{i}=j\right) \prod_{k=1}^{n} \eta_{k, j_{k}}
$$

where $\chi$ is the indicator function (i.e. $\chi\left(j_{i}=j\right)=1$ if proposition " $j_{i}=j$ " is true and $\chi\left(j_{i}=j\right)=0$ otherwise), so

$$
\begin{aligned}
& \sum_{i=1}^{n} \sum_{j=0}^{n^{\prime}} \frac{\partial \ln \zeta_{i, j}}{\partial x_{p}} P_{\mathrm{o}: \mathrm{o}}\left(A_{i, j} \cap C \mid C^{\prime}\right) \\
& =\Xi \sum_{i=1}^{n} \sum_{\substack{j_{1}=0 \\
j_{1} \notin X_{0}}}^{n^{\prime}} \sum_{j_{2} \notin X_{1}}^{n^{\prime}} \cdots \sum_{\substack{j_{n}=0 \\
j_{n} \notin X_{n-1}}}^{n^{\prime}} \sum_{j=0}^{n^{\prime}} \chi\left(j_{i}=j\right) \frac{\partial \ln \zeta_{i, j}}{\partial x_{p}} \prod_{k=1}^{n} \eta_{k, j_{k}} \\
& =\Xi \sum_{i=1}^{n} \sum_{\substack{j_{1}=0 \\
j_{1} \notin X_{0}}}^{n^{\prime}} \sum_{j_{2}=0}^{n_{2} \notin X_{1}} \ldots \sum_{\substack{j_{n}=0 \\
j_{n} \notin X_{n-1}}}^{n^{\prime}} \frac{\partial \ln \zeta_{i, j_{i}}}{\partial x_{p}} \prod_{k=1}^{n} \eta_{k, j_{k}} .
\end{aligned}
$$

If $j_{i}=0$, then $\eta_{i, j_{i}}=\zeta_{i, j_{i}}$; and if $j_{i} \neq 0$, the numerators of $\eta_{i, j_{i}}$ and $\zeta_{i, j_{i}}$ are the same and their denominators do not depend on $x_{p}$ : in all cases, $\partial \ln \eta_{i, j_{i}} / \partial x_{p}=\partial \ln \zeta_{i, j_{i}} / \partial x_{p}$. The righthand sides of Eqs. (76) and (78) are therefore identical. Dividing their lefthand sides by $P_{\text {o:o }}\left(C \mid C^{\prime}\right)$ and using Eqs. (74) and (4), we obtain, as announced,

$\frac{\partial \ln L_{\mathrm{o}: \mathrm{o}}}{\partial x_{p}}=\sum_{i=1}^{n} \sum_{j=0}^{n^{\prime}} \frac{\partial \ln \zeta_{i, j}}{\partial x_{p}} P_{\mathrm{o}: \mathrm{o}}\left(A_{i, j} \mid C \cap C^{\prime}\right)$.

For $x_{p}=f$ in particular, because of Eq. (37), and as under assumption $H_{\text {s:o }}$, Eq. (79) reduces to

$$
\frac{\partial \ln L_{\mathrm{o}: \mathrm{o}}}{\partial f}=\frac{n(1-f)-\sum_{i=1}^{n} P_{\mathrm{o}: \mathrm{o}}\left(A_{i, 0} \mid C \cap C^{\prime}\right)}{f(1-f)} \text {. }
$$

From Eq. (28), a maximum likelihood estimator of $f$ is thus

$$
\hat{f}_{\mathrm{o}: \mathrm{o}}=1-\frac{1}{n} \sum_{i=1}^{n} \hat{P}_{\mathrm{o}: \mathrm{o}}\left(A_{i, 0} \mid C \cap C^{\prime}\right)
$$

where $\hat{P}_{\text {o:o }}$ is the value of $P_{\text {o:o }}$ at $f=\hat{f}_{\text {o:o }}$.

To compare assumptions $H_{\mathrm{s}: \mathrm{o}}, H_{\mathrm{o}: 0}$, and $H_{\mathrm{s}: \mathrm{o}}$ and to select the most appropriate one to compute $P\left(A_{i, j} \mid C \cap C^{\prime}\right)$, an expression is needed for $L_{\mathrm{O}: 0}$. If probabilities $P_{\text {o:o }}\left(A_{i, 0} \mid C \cap C^{\prime}\right)$ are calculable, $L_{\mathrm{o} \text { :o }}$ may be obtained for any $f$ by integrating Eq. (80) with respect to $f$. Since all $K$ - and $K^{\prime}$-sources are unrelated and randomly distributed for $f=0$, the integration constant is (cf. Eq. (73))

$\left(\ln L_{\mathrm{o}: \mathrm{o}}\right)_{f=0}=\sum_{i=1}^{n} \ln \xi_{i, 0}+\sum_{j=1}^{n^{\prime}} \ln \xi_{0, j}$

\section{Practical implementation: the ASPECTS code}

\subsection{Overview}

To implement the results established in Sects. 3.1, 3.2, 4.1, and 4.2, we have built a Fortran 95 code, ASPECTS - a French acronym (pronounced [aspe] in International Phonetic Alphabet, not [æspekts]) for "Association PositionnellE/Probabilist $\mathbf{E}$ de 
CaTalogues de Sources", or "probabilistic positional association of source catalogs" in English. The source files are freely available ${ }^{4}$ at wWW2.iap.fr/users/fioc/Aspects/. The code compiles with IFORT and GFORTRAN.

Given two catalogs of sources with their positions and the uncertainties on these, ASPECTS computes, under assumptions $H_{\mathrm{s}: \mathrm{o}}, H_{\mathrm{o}: \mathrm{o}}$, and $H_{\mathrm{o}: \mathrm{s}}$, the overall likelihood $L$, estimates of $f$ and $f^{\prime}$, and the probabilities $P\left(A_{i, j} \mid C \cap C^{\prime}\right)$. It may also simulate all-sky catalogs for various association models (cf. Sect. 6.1).

We provide hereafter explanations of general interest for the practical implementation in AsPECTs of Eqs. (23), (39), (32), (71), (81), and (73). Some more technical points (such as the procedures used to search for nearby objects, simulate the positions of associated sources and integrate Eq. (80)) are only addressed in appendices to the documentation of the code (Fioc 2014). The latter also contains the following complements: another (but equivalent) expression for $L_{\mathrm{o}: \mathrm{o}}$, formulae derived under $H_{\mathrm{o}: \mathrm{s}}$, computations under $H_{\mathrm{o}: \mathrm{o}}$ for $n>n^{\prime}$, a calculation of the uncertainties on unknown parameters under $H_{\text {s:o }}$, and a proof of Eq. (41).

\subsection{Elimination of unlikely counterparts}

Under assumption $H_{\mathrm{s}: \mathrm{o}}$, computing the probability of association $P_{\text {s:o }}\left(A_{i, j} \mid C \cap C^{\prime}\right)$ between $M_{i}$ and $M_{j}^{\prime}$ from Eq. (23) is straightforward if $f$ and the positional uncertainties are known. However, the number of calculations for the whole sample or for determining $\hat{\boldsymbol{x}}$ is on the order of $n n^{\prime}$, a huge number for the catalogs available nowadays. We must therefore try to eliminate all unnecessary computations.

Since $\xi_{i, k}$ is given by a normal law if $i \neq 0$ and $k \neq 0$, it rapidly drops to almost 0 when we consider sources $M_{k}^{\prime}$ at increasing angular distance $\psi_{i, k}$ from $M_{i}$. Therefore, there is no need to compute $P_{\text {s:o }}\left(A_{i, j} \mid C \cap C^{\prime}\right)$ for all couples $\left(M_{i}, M_{j}^{\prime}\right)$ or to sum on all $k$ from 1 to $n^{\prime}$ in Eq. (24). More explicitly, let $R^{\prime}$ be some angular distance such that, for all $\left(M_{i}, M_{k}^{\prime}\right)$, if $\psi_{i, k} \geqslant R^{\prime}$ then $\xi_{i, k} \approx 0$, say

$$
R^{\prime} \gtrsim 5 \sqrt{\max _{\ell \in \llbracket 1, n \rrbracket} a_{\ell}^{2}+\max _{\ell \in \llbracket 1, n^{\prime} \rrbracket} a_{\ell}^{\prime 2}}
$$

where the $a_{\ell}$ and $a_{\ell}^{\prime}$ are the semi-major axes of the positional uncertainty ellipses of $K$ - and $K^{\prime}$-sources (cf. Appendix A.2.1; the square root in Eq. (83) is thus the maximal possible uncertainty on the relative position of associated sources). We may set $P_{\text {s:o }}\left(A_{i, j} \mid C \cap C^{\prime}\right)$ to 0 if $\psi_{i, j}>R^{\prime}$, and replace the sums $\sum_{k=1}^{n^{\prime}}$ by $\sum_{k=1 ; \psi_{i, k} \leqslant R^{\prime}}^{n^{\prime}}$ in Eq. (24): only nearby $K^{\prime}$-sources matter.

\subsection{Fraction of sources with a counterpart}

All the probabilities depend on $f$ and, possibly, on other unknown parameters like $\stackrel{\circ}{\sigma}$ and $\stackrel{\circ}{v}$ (cf. Appendices A.2.2 and A.2.3). Under assumption $H_{\mathrm{s}: \mathrm{o}}$, estimates of these parameters may be found by solving Eq. (28) using Eq. (33).

If the fraction of sources with a counterpart is the only unknown, the $\xi_{i, j}$ need to be computed only once and $\hat{f}_{\text {s:o }}$ may easily be determined from Eq. (39) by an iterative procedure.

\footnotetext{
4 Fortran 90 routines from Numerical Recipes (Press et al. 1992) are used to sort arrays and locate a value in an ordered table. Because of license constraints, we cannot provide them, but they may easily be replaced by free equivalents.
}

Denoting by $g$ the function

$g: f \in[0,1] \longmapsto 1-\frac{1}{n} \sum_{i=1}^{n} P_{\mathrm{s}: \mathrm{o}}\left(A_{i, 0} \mid C \cap C^{\prime}\right)$,

we now prove that, for any $\left.f_{0} \in\right] 0,1\left[\right.$, the sequence $\left(f_{k}\right)_{k \in \mathbb{N}}$ defined by $f_{k+1}:=g\left(f_{k}\right)$ tends to $\hat{f}_{\text {s:o }}$.

As is obvious from Eq. (24b), $P_{\mathrm{s}: 0}\left(A_{i, 0} \mid C \cap C^{\prime}\right)$ decreases for all $i$ when $f$ increases: $g$ is consequently an increasing function. Note also that, from Eqs. (38) and (84),

$g(f)=f+\frac{f(1-f)}{n} \frac{\partial \ln L_{\mathrm{s}: \mathrm{o}}}{\partial f}$.

The only fixed points of $g$ are thus 0,1 and the unique solution $\hat{f}_{\text {s:o }}$ to $\partial \ln L_{\text {s:o }} / \partial f=0$. Because $\partial^{2} \ln L_{\text {s:o }} / \partial f^{2}<0$ (cf. Eq. (41)) and $\hat{\partial} \ln L_{\mathrm{s}: \mathrm{o}} / \hat{\partial} f=0$, we have $\partial \ln L_{\mathrm{s}: \mathrm{o}} / \partial f \geqslant 0$ if $f \in\left[0, \hat{f}_{\mathrm{s}: \mathrm{o}}\right]$, so $g(f) \geqslant f$ in this interval by Eq. (85). Similarly, if $f \in\left[\hat{f}_{\text {s:o }}, 1\right]$, then $\partial \ln L_{\mathrm{s}: \mathrm{o}} / \partial f \leqslant 0$ and thus $g(f) \leqslant f$.

Consider the case $\left.\left.f_{0} \in\right] 0, \hat{f}_{\mathrm{s}: \mathrm{o}}\right]$. If $f_{k} \leqslant \hat{f}_{\mathrm{s}: 0}$, then as just shown, $g\left(f_{k}\right) \geqslant f_{k}$; we also have $g\left(f_{k}\right) \leqslant g\left(\hat{f}_{\text {s:o }}\right)=\hat{f}_{\text {s:o }}$, because $g$ is an increasing function and $\hat{f}_{\text {s:o }}$ is a fixed point of it. Since $g\left(f_{k}\right)=f_{k+1}$, the sequence $\left(f_{k}\right)_{k \in \mathbb{N}}$ is increasing and bounded from above by $\hat{f}_{\mathrm{s}: \mathrm{o}}$ : it therefore converges in $\left[f_{0}, \hat{f}_{\mathrm{s}: \mathrm{o}}\right]$. Because $g$ is continuous and $\hat{f}_{\text {s:o }}$ is the only fixed point in this interval, $\left(f_{k}\right)_{k \in \mathbb{N}}$ tends to $\hat{f}_{\mathrm{s}: o}$. Similarly, if $f_{0} \in\left[\hat{f}_{\mathrm{s}: \mathrm{o}}, 1\left[\right.\right.$, then $\left(f_{k}\right)_{k \in \mathbb{N}}$ is a decreasing sequence converging to $\hat{f}_{\text {s:o }}$.

Because of Eq. (81), this procedure also works in practice under assumption $H_{\mathrm{o}: \mathrm{o}}$ (with $P_{\text {s:o }}$ replaced by $P_{\text {o:o }}$ in Eq. (84)), although it is not obvious that $P_{\mathrm{o}: 0}\left(A_{i, 0} \mid C \cap C^{\prime}\right)$ decreases for all $i$ when $f$ increases, nor that $\partial^{2} \ln L_{\mathrm{o}: \mathrm{o}} / \partial f^{2}<0$. A good starting value $f_{0}$ may be $\hat{f}_{\text {s:o }}$.

\subsection{Computation of one-to-one probabilities of association}

What was said in Sect. 5.2 about eliminating unlikely counterparts in the calculation of probabilities under $H_{\text {s:o }}$ still holds under $H_{\text {o:o }}$. However, because of the combinatorial explosion of the number of terms in Eq. (71), computing $P_{\text {o:o }}\left(A_{i, j} \mid C \cap C^{\prime}\right)$ exactly is still clearly hopeless. Yet, after some wandering (Sects. 5.4.1 and 5.4.2), we found a working solution (Sect. 5.4.3).

\subsubsection{A first try}

Our first try was inspired by the (partially wrong) idea that, although all $K$-sources are involved in the numerator and denominator of Eq. (71), only those close to $M_{i}$ should matter in their ratio. A sequence of approximations converging to the true value of $P_{\mathrm{o}: \mathrm{o}}\left(A_{i, j} \mid C \cap C^{\prime}\right)$ might then be built as follows (all quantities defined or produced in this first try are written with the superscript "w" for "wrong").

To make things clear, consider $M_{1}$ and some possible counterpart $M_{j}^{\prime}$ within its neighborhood $\left(\psi_{1, j} \leqslant R^{\prime}\right)$ and assume that $M_{2}$ is the first nearest neighbor of $M_{1}$ in $K, M_{3}$ its second nearest neighbor, etc. For any $d \in \llbracket 1, n \rrbracket$, define

$$
p_{d}^{\mathrm{w}}(1, j):=\frac{\zeta_{1, j} \sum_{\substack{j_{2}=0 \\ j_{2} \notin X_{1}^{\star}}}^{n^{\prime}} \cdots \sum_{\substack{j_{d}=0 \\ j_{d} \notin X_{d-1}^{\star}}}^{n^{\prime}} \prod_{k=2}^{d} \eta_{k, j_{k}}^{\star}}{\sum_{\substack{j_{1}=0 \\ j_{1} \notin X_{0}}}^{n^{\prime}} \sum_{\substack{j_{2}=0 \\ j_{2} \notin X_{1}}}^{n^{\prime}} \cdots \sum_{\substack{j_{d}=0 \\ j_{d} \notin X_{d-1}}}^{n^{\prime}} \prod_{k=1}^{d} \eta_{k, j_{k}}} .
$$

The quantity $p_{d}^{\mathrm{w}}(1, j)$ thus depends only on $M_{1}$ and its $d-1$ nearest neighbors in $K$. As $p_{n}^{\mathrm{w}}(1, j)$ is the one-to-one probability of association between $M_{1}$ and $M_{j}^{\prime}$ (cf. Eq. (71)), the sequence $\left(p_{d}^{\mathrm{w}}[1, j]\right)$ tends to $P_{\mathrm{o}: \mathrm{o}}\left(A_{1, j} \mid C \cap C^{\prime}\right)$ when the depth $d$ 
of the recursive sums tends to $n$. After some initial fluctuations, $p_{d}^{\mathrm{w}}(1, j)$ enters a steady state. This occurs when $\psi\left(M_{1}, M_{d+1}\right)$ exceeds a distance $R$ equal to a few times $R^{\prime}$ (at least $2 R^{\prime}$ ). We may therefore think that the convergence is then achieved and stop the recursion at this $d$. It is all the more tempting that $p_{1}^{\mathrm{w}}(1, j)=P_{\mathrm{s}: \mathrm{o}}\left(A_{1, j} \mid C \cap C^{\prime}\right)$ and that the several-to-one probability looks like a first-order approximation to $P_{\text {o:o }} \ldots$

More formally and generally, for any $M_{i}$, let $\phi$ be a permutation on $K$ ordering the elements $M_{\phi(1)}, M_{\phi(2)}, \ldots, M_{\phi(n)}$ by increasing angular distance to $M_{i}$ (in particular, $M_{\phi(1)}=M_{i}$ ). For $j=0$ or $M_{j}^{\prime}$ within a distance $R^{\prime}$ (cf. Sect. 5.2) from $M_{i}$, and for any $d \in \llbracket 1, n \rrbracket$, define

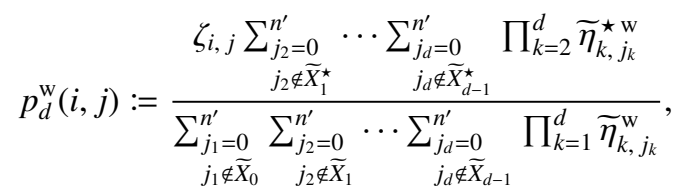

where, as in Eqs. (55), (67), (64), and (70),

$$
\left.\begin{array}{r}
\widetilde{X}_{k}:=X_{k} \quad \text { for all } k \in \llbracket 0, n \rrbracket ; \quad \widetilde{X}_{1}^{\star}:=\{j\} \backslash\{0\} ; \\
\widetilde{X}_{k}^{\star}:=\left(\widetilde{X}_{k-1}^{\star} \cup\left\{j_{k}\right\}\right) \backslash\{0\} \quad \text { for all } k \in \llbracket 2, n \rrbracket ; \\
\widetilde{\eta}_{k, 0}^{\mathrm{w}}:=\widetilde{\eta}_{k, 0}^{\star \mathrm{w}}:=\zeta_{\phi(k), 0} ; \quad \widetilde{\eta}_{k, j_{k}}^{\mathrm{w}}:=\frac{f \xi_{\phi(k), j_{k}}}{n^{\prime}-\# \widetilde{X}_{k-1}} \\
\quad \text { and } \widetilde{\eta}_{k, j_{k}}^{\star \mathrm{w}}:=\frac{f \xi_{\phi(k), j_{k}}}{n^{\prime}-\# \widetilde{X}_{k-1}^{\star}} \text { for } j_{k} \neq 0 .
\end{array}\right\}
$$

Let

$$
d_{\min }(i):=\min \left(d \in \llbracket 1, n \rrbracket \mid \psi\left[M_{i}, M_{\phi(d+1)}\right]>R\right) .
$$

Given above considerations, $P_{\mathrm{o}: \mathrm{o}}\left(A_{i, j} \mid C \cap C^{\prime}\right)$ can be evaluated as $p_{\mathrm{o}: \mathrm{o}}^{\mathrm{w}}(i, j):=p_{d_{\min }(i)}^{\mathrm{w}}(i, j)$.

The computation of $p_{d}^{\mathrm{w}}(i, j)$ may be further restricted (and in practice, because of the recursive sums in Eq. (87), must be) to sources $M_{j_{k}}^{\prime}$ in the neighborhood of the objects $\left(M_{\phi(k)}\right)_{k \in \llbracket 1, d \rrbracket}$, as explained in Sect. 5.2

\subsubsection{Failure of the first try}

To test the reliability of the evaluation of $P_{\mathrm{o}: \mathrm{o}}\left(A_{i, j} \mid C \cap C^{\prime}\right)$ by $p_{\mathrm{o}: \mathrm{o}}^{\mathrm{w}}(i, j)$, we simulated all-sky mock catalogs for one-to-one associations and analyzed them with a first version of ASPECTS. Simulations were run for $f=1 / 2, n^{\prime}=10^{5}, n \in \llbracket 10^{3}, 10^{5} \rrbracket$, and known circular positional uncertainties with $\stackrel{\circ}{\sigma}=10^{-3} \mathrm{rad}$ (see Sects. 6.1 and 6.2 for a detailed description).

Three estimators of $f$ were compared to the input value:

- $\hat{f}_{\mathrm{s}: \mathrm{o}}$, the value maximizing $L_{\mathrm{s}: \mathrm{o}}$ (Eq. (39));

- $\hat{f}_{\mathrm{o}: \mathrm{o}}^{\mathrm{w}}$, the value maximizing the one-to-one likelihood $L_{\mathrm{o}: \mathrm{o}}^{\mathrm{w}} \mathrm{de}-$ rived from the $p_{\mathrm{o}: 0}^{\mathrm{w}}$. This estimator is computed from Eq. (81) with $p_{\mathrm{o}: 0}^{\mathrm{w}}(i, 0)$ instead of $P_{\mathrm{o}: 0}\left(A_{i, 0} \mid C \cap C^{\prime}\right)$;

- $\hat{f}_{\mathrm{o} \text { s }}$, an estimator built from the one-to-several assumption in the following way: because $H_{\mathrm{o}: \mathrm{s}}$ is fully symmetric to $H_{\mathrm{s}: 0}$, we just need to swap $K$ and $K^{\prime}$ (i.e., swap $f$ and $f^{\prime}, n$ and $n^{\prime}$, etc.) in Eqs. (24), (26), and (39) to obtain $\hat{f}_{\mathrm{o}: \mathrm{s}}^{\prime}$ instead of $\hat{f}_{\mathrm{s}: o}$, and then, from Eq. $(42), \hat{f}_{\mathrm{o}: \mathrm{s}}$ instead of $\hat{f}_{\mathrm{s}: \mathrm{o}}^{\prime}$. The one-toseveral likelihood $L_{\mathrm{o}: \mathrm{s}}$ is computed from Eq. (32) in the same way.
The mean values of these estimators are plotted as a function of $n$ in Fig. 1a (error bars are smaller than the size of the points). As is obvious, the ad hoc estimator $\hat{f}_{\mathrm{o}: \mathrm{o}}^{\mathrm{w}}$ diverges from $f$ when $n$ increases. This statistical inconsistency ${ }^{5}$ seems surprising for a maximum likelihood estimator since the model on which it is based is correct by construction. However, all the demonstrations of consistency of maximum likelihood estimators we found in the literature (e.g., in Kendall \& Stuart 1979) rest on the assumption that the overall likelihood is the product of the probabilities of each datum, which is not the case for $L_{\mathrm{o} \text { o }}$ (cf. Eq. (73)). Since $\hat{f}_{\text {s:o }}$ is a good estimator of $f$, it might be used to compute $P_{\text {o:o }}\left(A_{i, j} \mid C \cap C^{\prime}\right)$ from $p_{\text {o:o }}^{\mathrm{w}}(i, j)-$ if the latter correctly approximates the former. By itself, the inconsistency of $\hat{f}_{\mathrm{o}: \mathrm{o}}^{\mathrm{w}}$ is therefore not a problem.

More embarrassing is that $H_{\mathrm{o}: \mathrm{o}}$ is not the most likely assumption (see Fig. 1b): the mean value of $\hat{L}_{\mathrm{o}: \mathrm{o}}^{\mathrm{w}}$ is less than that of $\hat{L}_{\mathrm{s}: \mathrm{o}}$ over the full interval of $n$ ! These two failures hint that the sequence $\left(p_{d}^{\mathrm{w}}[i, j]\right)$ has not yet converged to $P_{\mathrm{o}: \mathrm{o}}\left(A_{i, j} \mid C \cap C^{\prime}\right)$ at $d=d_{\min }(i)$.

To check this, we ran simulations with small numbers of sources ( $n$ and $n^{\prime}$ less than 10), so that we could compute $p_{n}^{\mathrm{w}}(i, j)$ exactly and study how $\left(p_{d}^{\mathrm{w}}[i, j]\right)$ tends to it. To test whether source confusion might be the reason for the problem, we created mock catalogs with very large positional uncertainties ${ }^{6} \stackrel{\circ}{\sigma}$, comparable to the distance between unrelated sources. Because the expressions given in Appendix A for $\xi_{i, j}$ are for planar normal laws and become wrong when the distance between $M_{i}$ and $M_{j}^{\prime}$ is more than a few degrees because of the curvature, we ran simulations on a whole circle instead of a sphere; nevertheless, we took $\stackrel{\circ}{ } \lesssim 30^{\circ}$ because the linear normal law is inappropriate on a circle for higher values, due to its finite extent. What we found is that, after the transient phase where it oscillates, $\left(p_{d}^{\mathrm{w}}[i, j]\right)$ slowly drifts to $P_{\mathrm{o}: \mathrm{o}}\left(A_{i, j} \mid C \cap C^{\prime}\right)$ and only converges at $d=n$ ! This drift was imperceptible for the high values of $n$ and $n^{\prime}$ used in Sect. 5.4.1.

\subsubsection{Reconsideration and solution}

To understand where the problem comes from, we consider the simplest case of interest: $n=n^{\prime}=2$. We assume moreover that $\xi_{1,2} \approx \xi_{2,1} \approx 0$. We then have

$$
\begin{aligned}
P_{\mathrm{o}: \mathrm{o}}\left(C \mid C^{\prime}\right) \approx\left([1-f]^{2} \xi_{1,0} \xi_{2,0}\right. & +\frac{[1-f] f}{2}\left[\xi_{1,0} \xi_{2,2}+\xi_{1,1} \xi_{2,0}\right] \\
& \left.+\frac{f^{2}}{2} \xi_{1,1} \xi_{2,2}\right) \mathrm{d}^{2} \boldsymbol{r}_{1} \mathrm{~d}^{2} \boldsymbol{r}_{2},
\end{aligned}
$$

$$
P_{\mathrm{o}: \mathrm{o}}\left(A_{1,0} \cap C \mid C^{\prime}\right) \approx(1-f) \xi_{1,0}\left([1-f] \xi_{2,0}+\frac{f}{2} \xi_{2,2}\right) \mathrm{d}^{2} \boldsymbol{r}_{1} \mathrm{~d}^{2} \boldsymbol{r}_{2},
$$

$$
P_{\mathrm{o}: \mathrm{o}}\left(A_{1,1} \cap C \mid C^{\prime}\right) \approx \frac{f}{2} \xi_{1,1}\left([1-f] \xi_{2,0}+f \xi_{2,2}\right) \mathrm{d}^{2} \boldsymbol{r}_{1} \mathrm{~d}^{2} \boldsymbol{r}_{2} .
$$

\footnotetext{
5 A consistent estimator is a statistic converging to the true value of a parameter when the size of the sample from which it is derived increases. The concept of consistency is not very clear in the context of this paper, since there are two sample sizes, $n$ and $n^{\prime}$.

6 Small positional uncertainties could also be used if sources were distributed on a small fraction of the sky, but there might be side effects.
} 
(a)

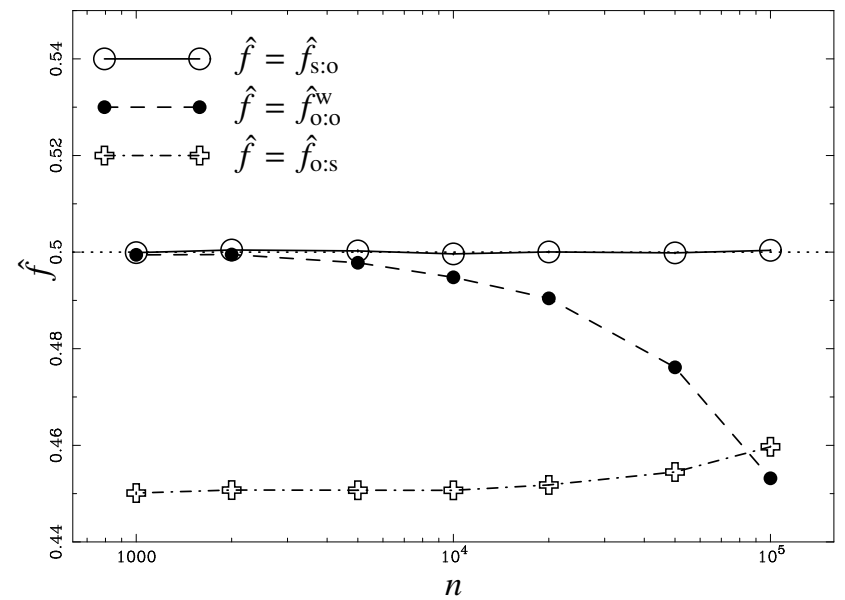

(b)

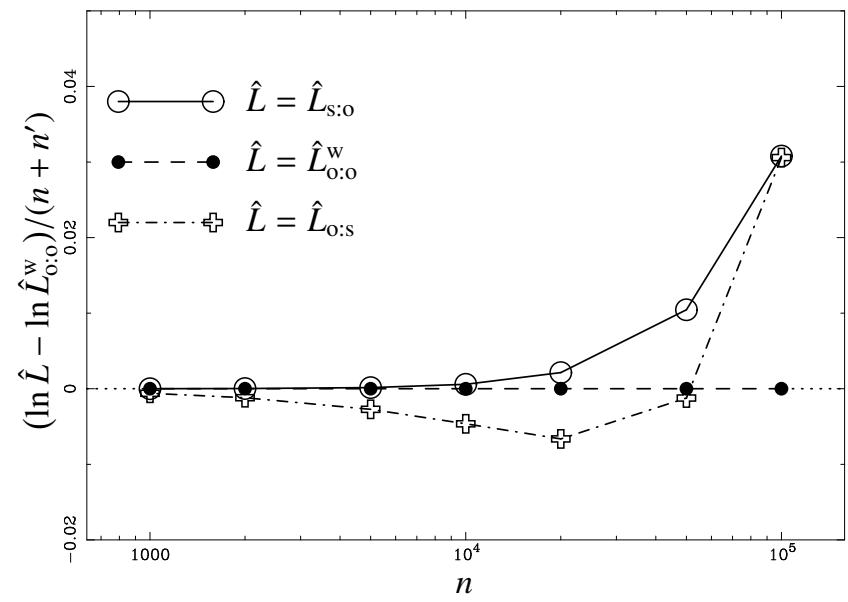

Fig. 1. One-to-one simulations for $f=1 / 2, n^{\prime}=10^{5}$, and circular positional uncertainty ellipses with $\stackrel{\circ}{\sigma}=10^{-3}$ rad (see Sects. 6.1 and 6.2 for details). a) Mean value of different estimators $\hat{f}$ of $f$ as a function of $n$. The dotted line indicates the input value of $f$. b) Normalized average maximum value $\hat{L}$ of different likelihoods as a function of $n$, compared to $\hat{L}_{\mathrm{o}: 0}^{\mathrm{w}}$.

The probabilities $P_{\mathrm{o}: \mathrm{o}}\left(A_{1, j} \mid C \cap C^{\prime}\right)=P_{\mathrm{o}: \mathrm{o}}\left(A_{1, j} \cap C \mid C^{\prime}\right) / P_{\mathrm{o}: \mathrm{o}}(C \mid$ $C^{\prime}$ ) obviously depend on $\xi_{2,2}$. In particular, if $\xi_{2,2} \ll \xi_{2,0}$,

$$
\left.\begin{array}{l}
P_{\mathrm{o}: \mathrm{o}}\left(A_{1,0} \mid C \cap C^{\prime}\right) \approx \frac{(1-f) \xi_{1,0}}{(1-f) \xi_{1,0}+f \xi_{1,1} / 2}, \\
P_{\mathrm{o}: \mathrm{o}}\left(A_{1,1} \mid C \cap C^{\prime}\right) \approx \frac{f \xi_{1,1} / 2}{(1-f) \xi_{1,0}+f \xi_{1,1} / 2} ;
\end{array}\right\}
$$

in that case, $P_{\mathrm{o}: \mathrm{o}}\left(A_{2,2} \mid C \cap C^{\prime}\right) \approx 0$, and both $M_{1}^{\prime}$ and $M_{2}^{\prime}$ are free for $M_{1}$. On the other hand, if $\xi_{2,2} \gg \xi_{2,0}$,

$$
\left.\begin{array}{l}
P_{\mathrm{o}: \mathrm{o}}\left(A_{1,0} \mid C \cap C^{\prime}\right) \approx \frac{(1-f) \xi_{1,0}}{(1-f) \xi_{1,0}+f \xi_{1,1} / 1}, \\
P_{\mathrm{o}: \mathrm{o}}\left(A_{1,1} \mid C \cap C^{\prime}\right) \approx \frac{f \xi_{1,1} / 1}{(1-f) \xi_{1,0}+f \xi_{1,1} / 1}
\end{array}\right\}
$$

in that case, $P_{\mathrm{o}: \mathrm{o}}\left(A_{2,2} \mid C \cap C^{\prime}\right) \approx 1: M_{2}$ and $M_{2}^{\prime}$ are almost certainly bound, so $M_{2}^{\prime}$ may not be associated to $M_{1}$, and $M_{1}^{\prime}$ is the only possible counterpart of $M_{1}$.

The difference between the results obtained for $\xi_{2,2} \ll \xi_{2,0}$ and $\xi_{2,2} \gg \xi_{2,0}$ shows that probabilities $P_{\mathrm{o}: \mathrm{o}}\left(A_{1, j} \mid C \cap C^{\prime}\right) \mathrm{de}-$ pend on the relative positions of $M_{2}$ and $M_{2}^{\prime}$, even when both $M_{2}$ and $M_{2}^{\prime}$ are distant from $M_{1}$ and $M_{1}^{\prime}$ : unlike the idea stated in Sect. 5.4.1, distant $K$-sources do matter for $P_{\text {o:o }}$ probabilities! However, as highlighted by the "/2" and "/1" factors in Eqs. (94) and (95), the distant $K$-source $M_{2}$ only changes the number of $K^{\prime}$-sources (two for $\xi_{2,2} \ll \xi_{2,0}$, one for $\xi_{2,2} \gg \xi_{2,0}$ ) that may be identified to $M_{1}$ : its exact position is unimportant.

This suggests the following solution: replace $n^{\prime}$ in Eq. (89) by the number $n_{\text {eff }}^{\prime}(i, d)$ of $K^{\prime}$-sources that may effectively be associated to $M_{i}$ and its $d-1$ nearest neighbors in $K$; i.e., dropping the superscript "w", define

$$
p_{d}(i, j):=\frac{\zeta_{i, j} \sum_{j_{2}=0}^{n^{\prime}} \cdots \sum_{j_{d}=0}^{n^{\prime}} \prod_{j_{d}} \prod_{\mathcal{X}_{d-1}^{\star}}^{\star}}{\prod_{k=2}^{d} \widetilde{\eta}_{k, j_{k}}^{\star}},
$$

where

$$
\left.\begin{array}{r}
\widetilde{\eta}_{k, 0}:=\widetilde{\eta}_{k, 0}^{\star}:=\zeta_{\phi(k), 0} ; \quad \widetilde{\eta}_{k, j_{k}}^{\star}:=\frac{f \xi_{\phi(k), j_{k}}}{n_{\mathrm{eff}}^{\prime}(i, d)-\# \widetilde{X}_{k-1}^{\star}} \\
\quad \text { and } \quad \widetilde{\eta}_{k, j_{k}}:=\frac{f \xi_{\phi(k), j_{k}}}{n_{\mathrm{eff}}^{\prime}(i, d)-\# \widetilde{X}_{k-1}} \quad \text { for } j_{k} \neq 0,
\end{array}\right\}
$$

and use $p_{\mathrm{o}: \mathrm{o}}(i, j):=p_{d_{\min }(i)}(i, j)$, where $d_{\min }(i)$ is defined by Eq. (90), to evaluate $P_{\mathrm{o}: \mathrm{o}}\left(A_{i, j} \mid C \cap C^{\prime}\right)$.

An estimate of $n_{\text {eff }}^{\prime}$ is given by ${ }^{7}$

$n_{\mathrm{eff}}^{\prime}(i, d)=n^{\prime}-\sum_{k=d+1}^{n}\left(1-P_{\mathrm{o}: \mathrm{o}}\left[A_{\phi(k), 0} \mid C \cap C^{\prime}\right]\right)$.

The sum in Eq. (98) is nothing but the typical number of counterparts in $K^{\prime}$ associated to distant $K$-sources. Note that $n_{\text {eff }}^{\prime}(i, d=$ $n)=n^{\prime}$, so we recover the theoretical result for $P_{\text {o:o }}\left(A_{i, j} \mid C \cap C^{\prime}\right)$ when all sources are considered. As $P_{\mathrm{o}: \mathrm{o}}$ depends on $n_{\mathrm{eff}}^{\prime}$ which in turn depends on $P_{\mathrm{o}: 0}$, both may be computed with a back and forth iteration; this procedure converges in a few steps if, instead of $P_{\mathrm{o}: 0}$, the value of $P_{\mathrm{s}: o}$ is taken to initiate the sequence.

\subsection{Tests of ASPECTS}

As computations made under assumption $H_{\mathrm{o}: \mathrm{o}}$ are complex (they involve recursive sums for instance), we made several consistency checks of the code. In particular, we swapped $K$ and $K^{\prime}$ for $n \neq n^{\prime}$ and compared quantities resulting from this swap (written with the superscript " $\leftrightarrow$ ") to original ones: within numerical errors, $\hat{f}_{\mathrm{o}: \mathrm{o}}^{\leftrightarrow}=\hat{f}_{\mathrm{o}: \mathrm{o}}$ and, for $f^{\prime \leftrightarrow}=f$, we get $L_{\mathrm{o}: \mathrm{o}}^{\leftrightarrow}=L_{\mathrm{o}: \mathrm{o}}$ and $P_{\mathrm{o}: \mathrm{o}}^{\leftrightarrow}\left(A_{j, i} \mid C^{\prime} \cap C\right)=P_{\mathrm{o}: \mathrm{o}}\left(A_{i, j} \mid C \cap C^{\prime}\right)$ for all $\left(M_{i}, M_{j}^{\prime}\right)$.

We moreover numerically checked for small $n$ and $n^{\prime}(\$ 5)$ that Eq. (73) and the integral of Eq. (80) with respect to $f$ are consistent and that ASPECTS returns the same value as MATHEMATICA (Wolfram 1996). For even smaller $n$ and $n^{\prime}(\leqslant 3)$, we confirmed that manual analytical expressions, obtained from the enumeration of all possible associations between $K$ and $K^{\prime}$, are identical to MATHEMATICA's symbolic calculations. For the large $n$ and $n^{\prime}$ of practical interest, although we did not give a formal proof of the solution of Sect. 5.4.3, the analysis of simulations (Sect. 6) makes us confident in the code.

7 Equation (98) is valid for any $f \in[0,1]$. When $f \approx \hat{f}_{\text {o:o }}$, it is more efficient to make the approximation $n_{\mathrm{eff}}^{\prime}(i, d) \approx n^{\prime}-f(n-d)$ : this expression accelerates the convergence to $\hat{f}_{\text {o:o }}$ of the sequence $\left(f_{k}\right)$ defined in Sect. 5.3. 
(a)

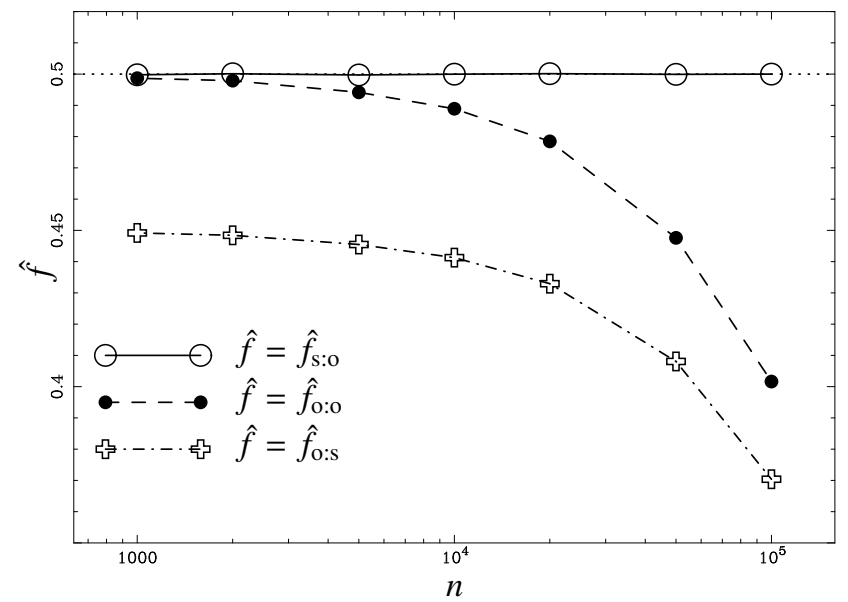

(b)

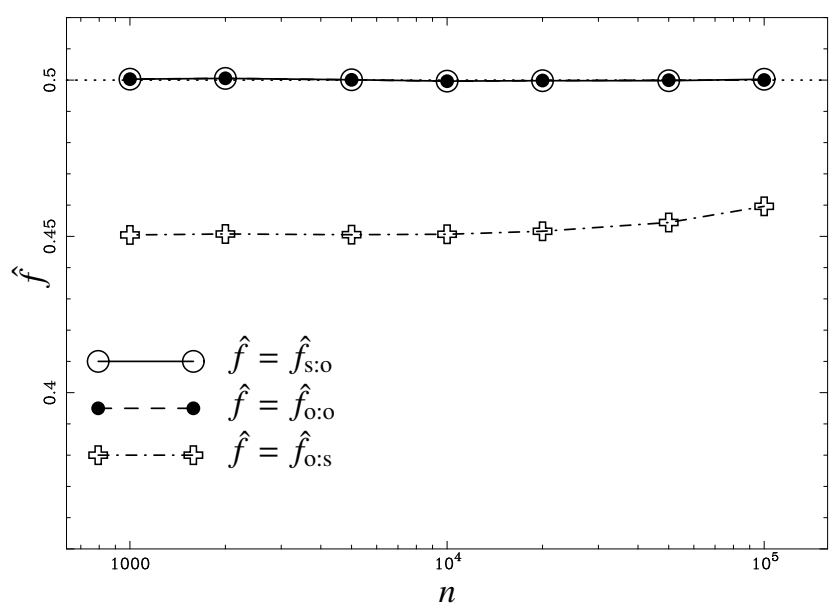

Fig. 2. Mean value of different estimators $\hat{f}$ of $f$ as a function of $n$ for $f=1 / 2$ (dotted line), $n^{\prime}=10^{5}$, and circular positional uncertainty ellipses with $\stackrel{\circ}{\sigma}=10^{-3} \mathrm{rad}$ (see Sects. 6.1 and 6.2 for details). a) Several-to-one simulations. b) One-to-one simulations $\left(\hat{f}_{\mathrm{s}: \mathrm{o}}\right.$ and $\hat{f}_{\mathrm{o}: \mathrm{o}}$ overlap).

\section{Simulations}

In this section, we analyze various estimators of the unknown parameters. Because of the complexity of the expressions we obtained, we did not try to do it analytically but used simulations. We also compare the likelihood of the assumptions $H_{\mathrm{s}: 0}, H_{\mathrm{o}: 0}$, and $H_{\mathrm{o}: \mathrm{s}}$, given the data.

\subsection{Creation of mock catalogs}

We have built all-sky mock catalogs with ASPECTS in the cases of several- and one-to-one associations. To do this, we first selected the indices of $f n$ objects in $K$, and associated randomly the index of a counterpart in $K^{\prime}$ to each of them; for one-to-one simulations, a given $K^{\prime}$-source was associated at most once. We then drew the true positions of $K^{\prime}$-sources uniformly on the sky. The true positions of $K$-sources without counterpart were also drawn in the same way; for sources with a counterpart, we took the true position of their counterpart. The observed positions of $K$ - and $K^{\prime}$-sources were finally computed from the true positions for given parameters $\left(a_{i}, b_{i}, \beta_{i}\right)$ and $\left(a_{j}^{\prime}, b_{j}^{\prime}, \beta_{j}^{\prime}\right)$ of the positional uncertainty ellipses (see Appendix A.2.1).

\subsection{Estimation of $f$ if positional uncertainty ellipses are known and circular}

Mock catalogs were created with $a_{i}=b_{i}=\sigma$ (see notations in Appendix A.2.1) for all $M_{i} \in K$ and with $a_{j}^{\prime}=b_{j}^{\prime}=\sigma^{\prime}$ for all $M_{j}^{\prime} \in K^{\prime}$. Positional uncertainty ellipses are therefore circular here. Only two parameters matter in that case: $f$ and

$\stackrel{\circ}{\sigma}:=\sqrt{\sigma^{2}+\sigma^{\prime 2}}$

Hundreds of simulations were run for $f=1 / 2, n^{\prime}=10^{5}$, $\stackrel{\circ}{\sigma}=10^{-3} \mathrm{rad}$, and $n \in \llbracket 10^{3}, 10^{5} \rrbracket$. We analyzed them with ASPECTS, knowing positional uncertainties, and plot the mean value of the estimators of $f$ listed in Sect. 5.4.2 as a function of $n$ in Fig. 2. This time, however, we replaced $\hat{f}_{\mathrm{o}: \mathrm{o}}^{\mathrm{w}}$ by the estimator $\hat{f}_{\mathrm{o}: \mathrm{o}}$ computed from the $p_{\mathrm{o}: \mathrm{o}}$.

For several-to-one simulations, $\hat{f}_{\mathrm{s}: \mathrm{o}}$ is by far the best estimator of $f$ and does not show any significant bias, whatever the value of $n$. Estimators $\hat{f}_{\mathrm{o}: \mathrm{o}}$ and $\hat{f}_{\mathrm{o} \text { : }}$ do not recover the input value of $f$, which is not surprising since they are not built from the right assumption here; moreover, while $\hat{f}_{\mathrm{s}: \mathrm{o}}, \hat{f}_{\mathrm{o}: \mathrm{s}}^{\prime}$, and $\hat{f}_{\mathrm{o}: \mathrm{o}}$ are obtained by maximizing $L_{\mathrm{s}: \mathrm{o}}, L_{\mathrm{o}: \mathrm{s}}$, and $L_{\mathrm{o}: \mathrm{o}}$, respectively, $\hat{f}_{\mathrm{o}: \mathrm{s}}$ is not directly fitted to the data.

For one-to-one simulations, and unlike $\hat{f}_{\mathrm{o}: 0}^{\mathrm{w}}, \hat{f}_{\mathrm{o}: \mathrm{o}}$ is a consistent estimator of $f$, as expected. Puzzlingly, $\hat{f}_{\text {s:o }}$ also works very well, maybe because $H_{\text {s:o }}$ is a more relaxed assumption than $H_{\mathrm{o}: 0}$; whatever the reason, this is not a problem.

\subsection{Simultaneous estimation of $f$ and $\stackrel{\circ}{\circ}$}

\subsubsection{Circular positional uncertainty ellipses}

How do different estimators of $f$ and $\stackrel{\circ}{\sigma}$ behave when the true values of positional uncertainties are also ignored? We show in Fig. 3 the result of simulations with the same input as in Sect. 6.2, except that $n=n^{\prime}=2 \times 10^{4}$. The likelihood $L_{\text {s:o }}$ peaks very close to the input value of $\boldsymbol{x}:=(f, \stackrel{\circ}{\sigma})$ for both types of simulations: $\hat{\boldsymbol{x}}_{\mathrm{s}: \mathrm{o}}$ is still an unbiased estimator of $\boldsymbol{x}$. For oneto-one simulations, $L_{\mathrm{o}: \mathrm{o}}$ is also maximal near the input value of $\boldsymbol{x}$, so $\hat{\boldsymbol{x}}_{\mathrm{o}: \mathrm{o}}$ is unbiased, too.

\subsubsection{Elongated positional uncertainty ellipses}

To test the robustness of estimators of $f$, we ran simulations with the same parameters, but with elongated positional uncertainty ellipses: we took $a_{i}=a_{j}^{\prime}=1.5 \times 10^{-3} \mathrm{rad}$ and $b_{i}=b_{j}^{\prime}=a_{i} / 3$ for all $\left(M_{i}, M_{j}^{\prime}\right) \in K \times K^{\prime}$. These ellipses were randomly oriented; i.e., position angles (cf. Appendix A.2.1) $\beta_{i}$ and $\beta_{j}^{\prime}$ have uniform random values in $[0, \pi[$. We then estimated $f$, but ignoring these positional uncertainties (see Fig. 4).

Although the model from which the parameters are fitted is inaccurate here (the $\xi_{i, j}$ are computed assuming circular positional uncertainties instead of the unknown elliptical ones), the input value of $f$ is still recovered by $\hat{f}_{\text {s:o }}$ for both types of simulations and by $\hat{f}_{\text {o:o }}$ for one-to-one simulations. The fitting also provides the typical positional uncertainty $\stackrel{\circ}{\sigma}$ on the relative positions of associated sources.

\subsection{Choice of association model}

Now, given the two catalogs, which assumption should we adopt to compute the probabilities $P\left(A_{i, j} \mid C \cap C^{\prime}\right)$ : several-to-one, 
M. Fioc: Probabilistic positional association of catalogs of astrophysical sources: the ASPECTS code

(a)

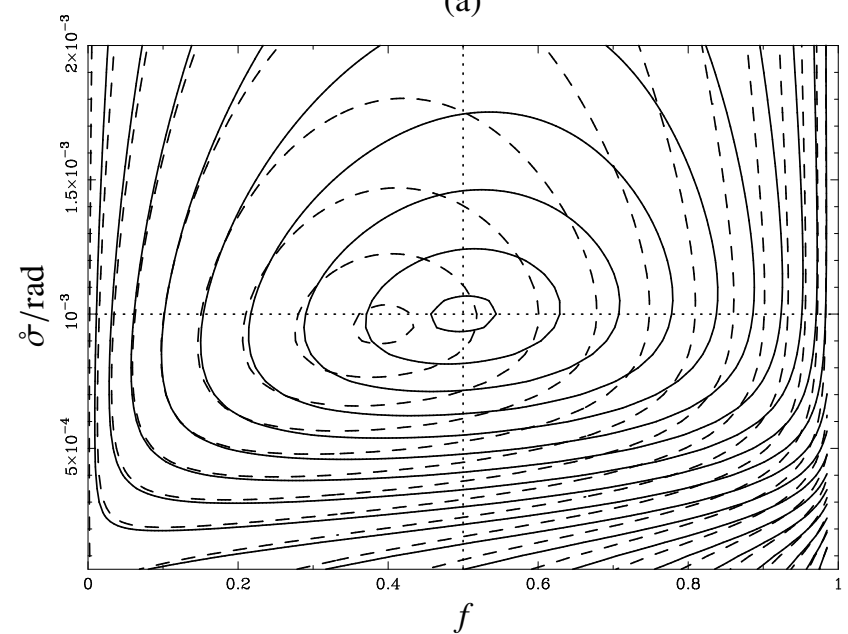

(b)

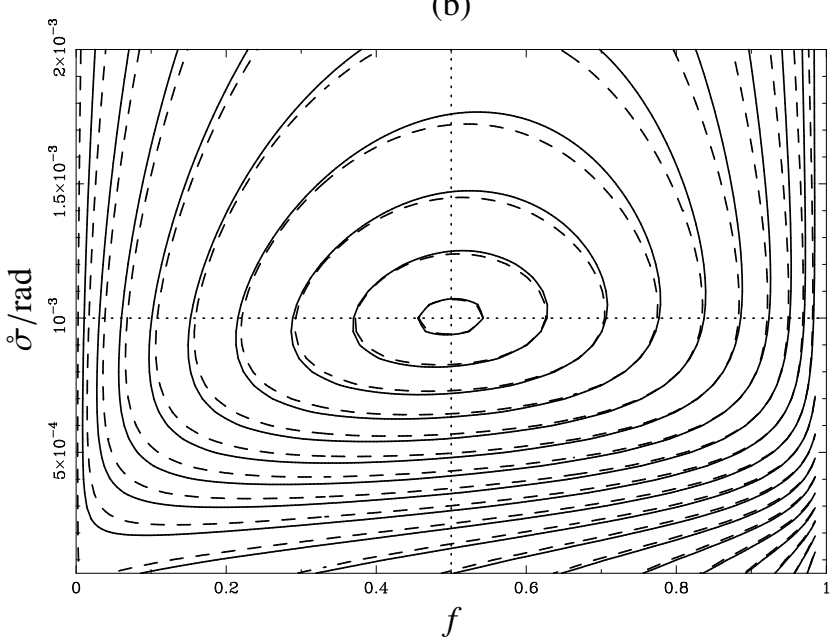

Fig. 3. Contour lines of $L_{\mathrm{s}: \mathrm{o}}$ (solid) and $L_{\mathrm{o}: \mathrm{o}}$ (dashed) in the $(f, \stackrel{\circ}{\sigma})$ plane. Input parameters are the same as in Fig. 2 , except that $n=n^{\prime}=2 \times 10^{4}$; the input values of $f$ and $\stackrel{\circ}{\sigma}$ are indicated by dotted lines (see Sect. 6.3.1 for details). a) Several-to-one simulations. b) One-to-one simulations.

(a)

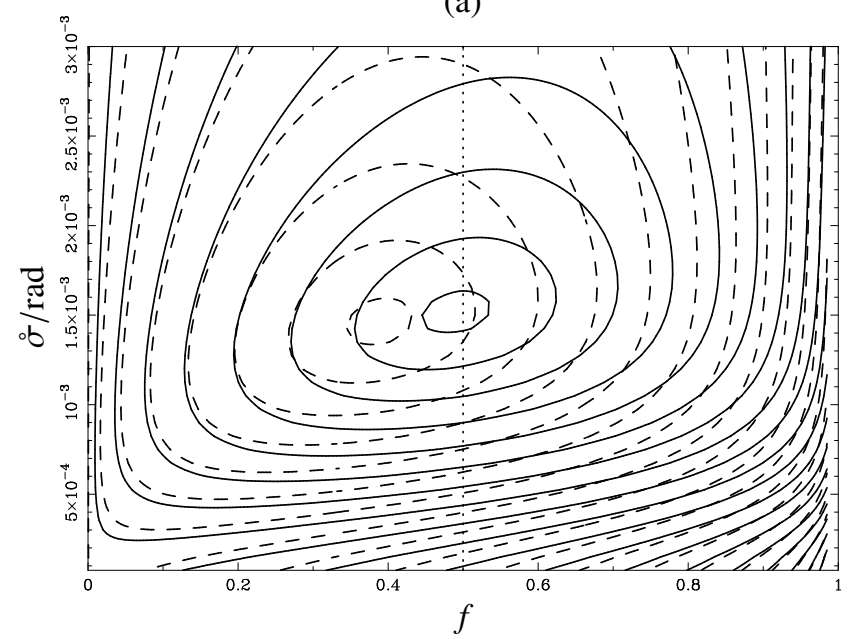

(b)

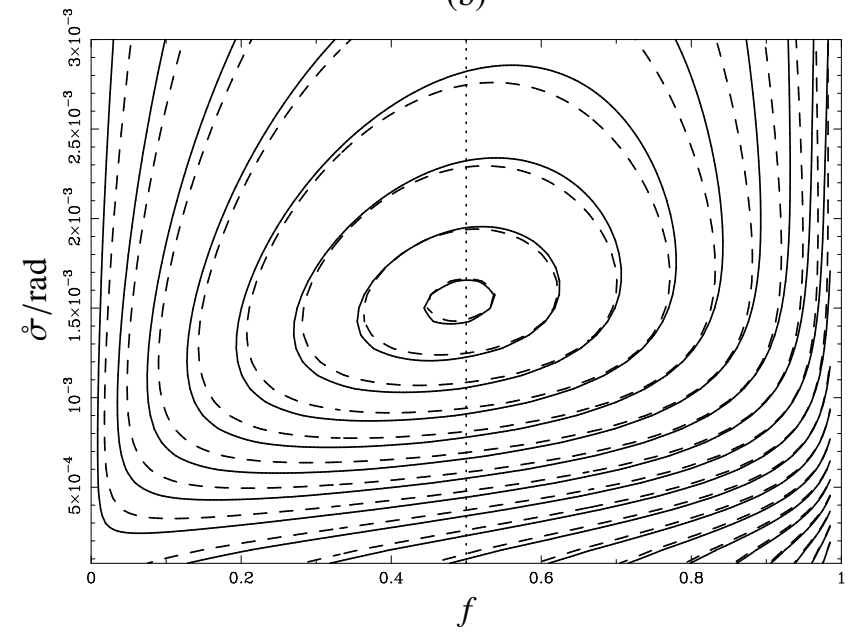

Fig. 4. Contour lines of $L_{\text {s:o }}$ (solid) and $L_{\mathrm{o}: \mathrm{o}}$ (dashed) in the $(f, \stackrel{\circ}{\sigma})$ plane. Input parameters are the same as in Fig. 2, except that positional uncertainty ellipses are elongated and randomly oriented (see Sect. 6.3.2 for details); the input value of $f$ is indicated by a dotted line. a) Several-to-one simulations. b) One-to-one simulations.

one-to-one or one-to-several? As shown in Fig. 5, for known positional uncertainties and a given $n^{\prime}$, source confusion is rare at low values of $n$ (there is typically at most one possible counterpart) and all assumptions are equally likely. At larger $n, \hat{L}_{\mathrm{s}: \mathrm{o}}>$ $\hat{L}_{\mathrm{o}: \mathrm{o}}>\hat{L}_{\mathrm{o}: \mathrm{s}}$ for several-to-one simulations; as expected, for oneto-one simulations, $\hat{L}_{\mathrm{o}: \mathrm{o}}>\hat{L}_{\mathrm{s}: \mathrm{o}}$ and $\hat{L}_{\mathrm{o}: \mathrm{o}}>\hat{L}_{\mathrm{o}: \mathrm{s}}$, with $\hat{L}_{\mathrm{s}: \mathrm{o}} \approx \hat{L}_{\mathrm{o}: \mathrm{s}}$ for $n=n^{\prime}$. In all cases, on average, the right assumption is the most likely. This is also true when positional uncertainties are ignored (Sect. 6.3).

The calculation of $L_{\mathrm{o} \text { :o }}$ is lengthy, and as a substitute to the comparison of the likelihoods, the following procedure may be applied to select the most appropriate assumption to compute the probabilities of association: if $\hat{f}_{\mathrm{s}: \mathrm{o}} n \approx \hat{f}_{\mathrm{o}: \mathrm{s}}^{\prime} n^{\prime}$, use $H_{\mathrm{o}: \mathrm{o}}$; if $\hat{f}_{\mathrm{s}: \mathrm{o}} n \not \hat{f}_{\mathrm{o}: \mathrm{s}}^{\prime} n^{\prime}$, then use $H_{\mathrm{s}: \mathrm{o}}$ if $\hat{f}_{\mathrm{s}: \mathrm{o}} n>\hat{f}_{\mathrm{o}: \mathrm{s}}^{\prime} n^{\prime}$, and $H_{\mathrm{o}: \mathrm{s}}$ otherwise.

\section{Conclusion}

In this paper, we computed the probabilities of positional association of sources between two catalogs $K$ and $K^{\prime}$ under two different assumptions: first, the easy case where several $K$-objects may share the same counterpart in $K^{\prime}$, then the more natural but numerically intensive case of one-to-one associations only between $K$ and $K^{\prime}$.

These probabilities depend on at least one unknown parameter: the fraction of sources with a counterpart. If the positional uncertainties are unknown, other parameters are required to compute the probabilities. We calculated the likelihood of observing all the $K$ - and $K^{\prime}$-sources at their effective positions under each of the two assumptions described above, and estimated the unknown parameters by maximizing these likelihoods. The latter are also used to select the best association model.

These relations were implemented in a code, AsPECTS, which we make public and with which we analyzed all-sky several-to-one and one-to-one simulations. In all cases, the assumption with the highest likelihood is the right one, and estimators of unknown parameters obtained for it do not show any bias.

In the simulations, we assumed that the density of $K$ - and $K^{\prime}$-sources was uniform on the sky area $S$ : the quantities $\xi_{i, 0}$ and $\xi_{0, j}$ used to compute the probabilities are then equal to $1 / S$. If the density of objects is not uniform, we might take $\xi_{i, 0}=\rho\left(M_{i}\right) / n$ and $\xi_{0, j}=\rho^{\prime}\left(M_{j}^{\prime}\right) / n^{\prime}$, where $\rho$ and $\rho^{\prime}$ are, respectively, the local 
(a)

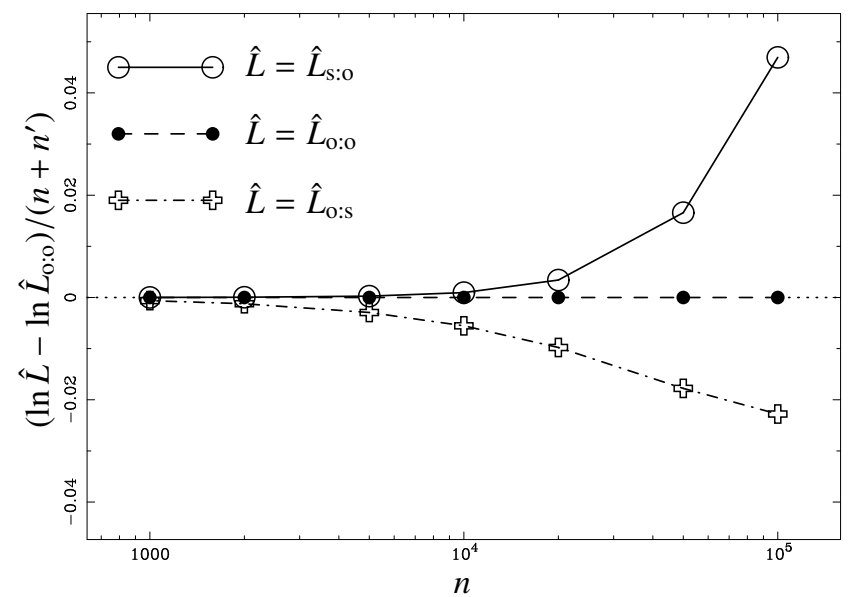

(b)

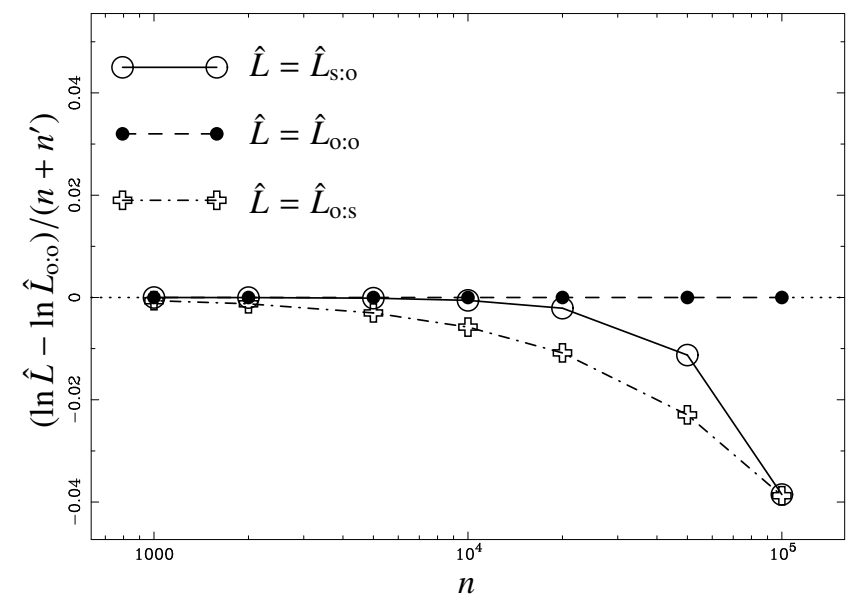

Fig. 5. Normalized average maximum value $\hat{L}$ of different likelihoods as a function of $n$, compared to $\hat{L}_{\text {o:o }}$. Simulations are the same as in Fig. 2 . a) Several-to-one simulations. b) One-to-one simulations.

surface densities of $K$ - and $K^{\prime}$-sources; but if the $\rho^{\prime} / \rho$ ratio varies on the sky, so will the fraction of sources with a counterpart something we did not try to model. Considering clustering or the side effects ${ }^{8}$ due to a small $S$, as well as taking priors on the SED of objects into account was also beyond the scope of this paper.

In spite of these limitations, ASPECTS is a robust tool that should help astronomers cross-identify astrophysical sources automatically, efficiently and reliably.

\section{Appendix A: Probability distribution of the observed relative positions of associated sources}

\section{A.1. Properties of normal laws}

We first recall a few standard results. The probability that an $m$ dimensional normally distributed random vector $\boldsymbol{W}$ of mean $\boldsymbol{\mu}$ and variance $\Gamma$ falls in some domain $\Omega$ is

$P(\boldsymbol{W} \in \Omega)=\int_{\boldsymbol{w} \in \Omega} \frac{\exp \left(-\frac{1}{2}[\boldsymbol{w}-\boldsymbol{\mu}]_{B}^{\top} \cdot \Gamma_{B}^{-1} \cdot[\boldsymbol{w}-\boldsymbol{\mu}]_{B}\right)}{(2 \pi)^{m / 2} \sqrt{\operatorname{det} \Gamma_{B}}} \mathrm{~d}^{m} \boldsymbol{w}_{B}$,

where $B:=\left(\boldsymbol{u}_{1}, \ldots, \boldsymbol{u}_{m}\right)$ is a basis, $\boldsymbol{w}$ is a vector, $\boldsymbol{w}_{B}=$ $\left(w_{1}, \ldots, w_{m}\right)^{\top}$ (resp. $\left.\boldsymbol{\mu}_{B}\right)$ is the column vector expression of $\boldsymbol{w}$ (resp. $\boldsymbol{\mu}$ ) in $B, \mathrm{~d}^{m} \boldsymbol{w}_{B}:=\prod_{i=1}^{m} \mathrm{~d} w_{i}$, and $\Gamma_{B}$ is the covariance matrix of $W$ (i.e. the matrix representation of $\Gamma$ ) in $B$. We denote this by $\boldsymbol{W} \sim G_{m}(\boldsymbol{\mu}, \Gamma)$.

In another basis $B^{\prime}:=\left(\boldsymbol{u}_{1}^{\prime}, \ldots, \boldsymbol{u}_{m}^{\prime}\right)$, we have $\boldsymbol{w}_{B}=T_{B \rightarrow B^{\prime}} \cdot \boldsymbol{w}_{B^{\prime}}$, where $T_{B \rightarrow B^{\prime}}$ is the transformation matrix from $B$ to $B^{\prime}$ (i.e. $\boldsymbol{u}_{j}^{\prime}=$ $\left.\sum_{i=1}^{m}\left(T_{B \rightarrow B^{\prime}}\right)_{i, j} \boldsymbol{u}_{i}\right)$. Since $\mathrm{d}^{m} \boldsymbol{w}_{B}=\left|\operatorname{det} T_{B \rightarrow B^{\prime}}\right| \mathrm{d}^{m} \boldsymbol{w}_{B^{\prime}}$ and

$$
\begin{aligned}
& (\boldsymbol{w}-\boldsymbol{\mu})_{B}^{\top} \cdot \Gamma_{B}^{-1} \cdot(\boldsymbol{w}-\boldsymbol{\mu})_{B} \\
& \quad=(\boldsymbol{w}-\boldsymbol{\mu})_{B^{\prime}}^{\top} \cdot\left(T_{B \rightarrow B^{\prime}}^{-1} \cdot \Gamma_{B} \cdot\left[T_{B \rightarrow B^{\prime}}^{-1}\right]^{\top}\right)^{-1} \cdot(\boldsymbol{w}-\boldsymbol{\mu})_{B^{\prime}},
\end{aligned}
$$

we still obtain

$P(\boldsymbol{W} \in \Omega)=\int_{\boldsymbol{w} \in \Omega} \frac{\exp \left(-\frac{1}{2}[\boldsymbol{w}-\boldsymbol{\mu}]_{B^{\prime}}^{\top} \cdot \Gamma_{B^{\prime}}^{-1} \cdot[\boldsymbol{w}-\boldsymbol{\mu}]_{B^{\prime}}\right)}{(2 \pi)^{m / 2} \sqrt{\operatorname{det} \Gamma_{B^{\prime}}}} \mathrm{d}^{m} \boldsymbol{w}_{B^{\prime}}$,

\footnotetext{
8 The impact of clustering or of side effects on estimators of unknown parameters might however easily be tested through simulations.
}

where $\Gamma_{B^{\prime}}:=T_{B \rightarrow B^{\prime}}^{-1} \cdot \Gamma_{B} \cdot\left(T_{B \rightarrow B^{\prime}}^{-1}\right)^{\top}$ is the covariance matrix of $\boldsymbol{W}$ in $B^{\prime}$. In the following, $B$ and $B^{\prime}$ are orthonormal bases, so $T_{B \rightarrow B^{\prime}}$ is a rotation matrix. From $T_{B \rightarrow B^{\prime}}^{\top}=T_{B \rightarrow B^{\prime}}^{-1}$, we get

$\Gamma_{B^{\prime}}=T_{B \rightarrow B^{\prime}}^{\top} \cdot \Gamma_{B} \cdot T_{B \rightarrow B^{\prime}}$.

For independent random vectors $\boldsymbol{W}_{1} \sim G_{m}\left(\boldsymbol{\mu}_{1}, \Gamma_{1}\right)$ and $\boldsymbol{W}_{2} \sim$ $G_{m}\left(\mu_{2}, \Gamma_{2}\right)$, we have

$\boldsymbol{W}_{1} \pm \boldsymbol{W}_{2} \sim G_{m}\left(\boldsymbol{\mu}_{1} \pm \boldsymbol{\mu}_{2}, \Gamma_{1}+\Gamma_{2}\right)$.

\section{A.2. Covariance matrix of the probability distribution of relative positions}

We now use these results to derive the probability distribution of vector $\boldsymbol{r}_{i, j}:=\boldsymbol{r}_{j}^{\prime}-\boldsymbol{r}_{i}$, where $\boldsymbol{r}_{i}$ and $\boldsymbol{r}_{j}^{\prime}$ are, respectively, the observed positions of source $M_{i}$ of $K$ and of its counterpart $M_{j}^{\prime}$ in $K^{\prime}$. Introducing the true positions $\boldsymbol{r}_{0, i}$ and $\boldsymbol{r}_{0, j}^{\prime}$ of $M_{i}$ and $M_{j}^{\prime}$, we have

$\boldsymbol{r}_{i, j}=\left(\boldsymbol{r}_{j}^{\prime}-\boldsymbol{r}_{0, j}^{\prime}\right)+\left(\boldsymbol{r}_{0, j}^{\prime}-\boldsymbol{r}_{0, i}\right)+\left(\boldsymbol{r}_{0, i}-\boldsymbol{r}_{i}\right)$.

\section{A.2.1. Covariance matrix for identical true positions and known positional uncertainties}

Assume $^{9}$, as is usual, that

$\boldsymbol{r}_{i}-\boldsymbol{r}_{0, i} \sim G_{2}\left(\mathbf{0}, \Gamma_{i}\right) \quad$ and $\quad \boldsymbol{r}_{j}^{\prime}-\boldsymbol{r}_{0, j}^{\prime} \sim G_{2}\left(\mathbf{0}, \Gamma_{j}^{\prime}\right)$.

If the true positions of $M_{i}$ and $M_{j}^{\prime}$ are identical (case of point sources), then, from Eqs. (A.5)-(A.7),

$\boldsymbol{r}_{i, j} \sim G_{2}\left(\mathbf{0}, \Gamma_{i, j}\right), \quad$ where $\Gamma_{i, j}:=\Gamma_{i}+\Gamma_{j}^{\prime}$.

(See also Condon et al. 1995.) In Eqs. (A.7), $\boldsymbol{r}_{i}-\boldsymbol{r}_{0, i}$ and $\boldsymbol{r}_{j}^{\prime}-\boldsymbol{r}_{0, j}^{\prime}$ must be considered as the projections (gnomonic ones, for instance) of these vectors on the planes tangent to the sphere at $M_{i}$ and $M_{j}^{\prime}$, respectively; Eqs. (A.7) are approximations, valid only

\footnotetext{
9 None of the results established outside of Appendix A depends on this assumption.
} 
because positional uncertainties are small ${ }^{10}$. Equation (A.8) is also an approximation: it is appropriate because the observed positions of associated sources $M_{i}$ and $M_{j}^{\prime}$ are close, so the tangent planes to the sphere at both points nearly coincide.

To use Eq. (A.8), we now compute the column vector expression of $\boldsymbol{r}_{i, j}$ and the covariance matrices associated to $\Gamma_{i}, \Gamma_{j}^{\prime}$, and $\Gamma_{i, j}$ in some common basis. For convenience, we drop the subscript and the "prime" symbol in the following whenever an expression only depends on either $M_{i}$ or $M_{j}^{\prime}$.

Let $\left(\boldsymbol{u}_{x}, \boldsymbol{u}_{y}, \boldsymbol{u}_{z}\right)$ be a direct orthonormal basis, with $\boldsymbol{u}_{z}$ oriented from the Earth's center $O$ to the North Celestial Pole and $\boldsymbol{u}_{x}$ from $O$ to the Vernal Point. At a point $M$ of right ascension $\alpha$ and declination $\delta$, a direct orthonormal basis $\left(\boldsymbol{u}_{r}, \boldsymbol{u}_{\alpha}, \boldsymbol{u}_{\delta}\right)$ is defined by

$$
\begin{aligned}
& \boldsymbol{u}_{r}:=\frac{\boldsymbol{O M}}{\|\boldsymbol{O M}\|}=\cos \delta \cos \alpha \boldsymbol{u}_{x}+\cos \delta \sin \alpha \boldsymbol{u}_{y}+\sin \delta \boldsymbol{u}_{z} \\
& \boldsymbol{u}_{\alpha}:=\frac{\partial \boldsymbol{u}_{r} / \partial \alpha}{\left\|\partial \boldsymbol{u}_{r} / \partial \alpha\right\|}=-\sin \alpha \boldsymbol{u}_{x}+\cos \alpha \boldsymbol{u}_{y} \\
& \boldsymbol{u}_{\delta}:=\frac{\partial \boldsymbol{u}_{r} / \partial \delta}{\left\|\partial \boldsymbol{u}_{r} / \partial \delta\right\|}=-\sin \delta \cos \alpha \boldsymbol{u}_{x}-\sin \delta \sin \alpha \boldsymbol{u}_{y}+\cos \delta \boldsymbol{u}_{z}
\end{aligned}
$$

The uncertainty ellipse on the position of $M$ is characterized by the lengths $a$ and $b$ of its semi-major and semi-minor axes, and by the position angle $\beta$ between the north and the semi-major axis. Let $\boldsymbol{u}_{a}$ and $\boldsymbol{u}_{b}$ be unit vectors directed along the major and the minor axes, respectively, and such that $\left(\boldsymbol{u}_{r}, \boldsymbol{u}_{a}, \boldsymbol{u}_{b}\right)$ is a direct orthonormal basis and that $\beta:=\angle\left(\boldsymbol{u}_{\delta}, \boldsymbol{u}_{a}\right)$ is in $[0, \pi[$ when counted eastward. Since $\left(\boldsymbol{u}_{\alpha}, \boldsymbol{u}_{\delta}\right)$ is obtained from $\left(\boldsymbol{u}_{a}, \boldsymbol{u}_{b}\right)$ by a $(\beta-\pi / 2)$-counterclockwise rotation in the plane oriented by $+\boldsymbol{u}_{r}$, we have $T_{\left(\boldsymbol{u}_{a}, \boldsymbol{u}_{b}\right) \rightarrow\left(\boldsymbol{u}_{\alpha}, \boldsymbol{u}_{\delta}\right)}=\operatorname{Rot}(\beta-\pi / 2)$, where, for any angle $\tau$,

$\operatorname{Rot} \tau:=\left(\begin{array}{cc}\cos \tau & -\sin \tau \\ \sin \tau & \cos \tau\end{array}\right)$.

Using notation

$\operatorname{Diag}\left(d_{1}, d_{2}\right):=\left(\begin{array}{cc}d_{1} & 0 \\ 0 & d_{2}\end{array}\right)$

for diagonal matrices, we have ${ }^{11} \Gamma_{\left(\boldsymbol{u}_{a}, \boldsymbol{u}_{b}\right)}=\operatorname{Diag}\left(a^{2}, b^{2}\right)$ and

$\Gamma_{\left(\boldsymbol{u}_{\alpha}, \boldsymbol{u}_{\delta}\right)}=\operatorname{Rot}^{\top}(\beta-\pi / 2) \cdot \operatorname{Diag}\left(a^{2}, b^{2}\right) \cdot \operatorname{Rot}(\beta-\pi / 2)$.

As noticed by Pineau et al. (2011), around the Poles, even for sources $M_{i}$ and $M_{j}^{\prime}$ close to each other, we may have $\left(\boldsymbol{u}_{\alpha, i}, \boldsymbol{u}_{\delta, i}\right) \not$ $\left(\boldsymbol{u}_{\alpha^{\prime}, j}, \boldsymbol{u}_{\delta^{\prime}, j}\right)$ : the covariance matrices $\left(\Gamma_{i}\right)_{\left(\boldsymbol{u}_{\alpha, i}, \boldsymbol{u}_{\delta, i}\right)}$ and $\left(\Gamma_{j}^{\prime}\right)_{\left(\boldsymbol{u}_{\alpha^{\prime}, j}, \boldsymbol{u}_{\delta^{\prime}, j}\right)}$ must therefore be first converted to a common basis before their summation in Eq. (A.8). We use the same basis as Pineau et al. (2011), denoted by $(\boldsymbol{t}, \boldsymbol{n})$ below. While the results we get are intrinsically the same, some people may find our expressions more convenient.

Denote by $\boldsymbol{n}:=\boldsymbol{u}_{r, i} \times \boldsymbol{u}_{r^{\prime}, j} /\left\|\boldsymbol{u}_{r, i} \times \boldsymbol{u}_{r^{\prime}, j}\right\|$ a unit vector perpendicular to the plane $\left(O, M_{i}, M_{j}^{\prime}\right)$. Because $\psi_{i, j}:=\angle\left(\boldsymbol{u}_{r, i}, \boldsymbol{u}_{r^{\prime}, j}\right) \in$

\footnotetext{
${ }^{10}$ If it were not the case, the probability of $\boldsymbol{r}_{i}-\boldsymbol{r}_{0, i}$ and $\boldsymbol{r}_{j}^{\prime}-\boldsymbol{r}_{0, j}^{\prime}$ might be modeled using Kent (1982) distributions (an adaptation to the sphere of the planar normal law), but no result like Eq. (A.8) would then hold: unlike Gaussians, Kent distributions are not stable.

${ }^{11}$ We seize this opportunity to correct Eqs. (A.8) to (A.11) of Pineau et al. (2011): $a$ and $b$ should be replaced by their squares in these formulae.
}

$[0, \pi]$, we have $\boldsymbol{u}_{r, i} \cdot \boldsymbol{u}_{r^{\prime}, j}=\cos \psi_{i, j}$ and $\left\|\boldsymbol{u}_{r, i} \times \boldsymbol{u}_{r^{\prime}, j}\right\|=\sin \psi_{i, j}$, so

$\psi_{i, j}=\arccos \left(\cos \delta_{i} \cos \delta_{j}^{\prime} \cos \left[\alpha_{j}^{\prime}-\alpha_{i}\right]+\sin \delta_{i} \sin \delta_{j}^{\prime}\right)$,

and

$\boldsymbol{n}=\frac{\boldsymbol{u}_{r, i} \times \boldsymbol{u}_{r^{\prime}, j}}{\sin \psi_{i, j}}$.

Let $\gamma_{i}:=\angle\left(\boldsymbol{n}, \boldsymbol{u}_{\delta, i}\right)$ and $\gamma_{j}^{\prime}:=\angle\left(\boldsymbol{n}, \boldsymbol{u}_{\delta^{\prime}, j}\right)$ be angles oriented clockwise around $+\boldsymbol{u}_{r, i}$ and $+\boldsymbol{u}_{r^{\prime}, j}$, respectively. Angle $\gamma_{i}$ is fully determined by the following expressions (cf. Eqs. (A.16) and (A.9)-(A.11)):

$$
\begin{aligned}
\cos \gamma_{i} & =\boldsymbol{n} \cdot \boldsymbol{u}_{\delta, i}=\frac{\left(\boldsymbol{u}_{r, i} \times \boldsymbol{u}_{r^{\prime}, j}\right) \cdot \boldsymbol{u}_{\delta, i}}{\sin \psi_{i, j}}=\frac{\left(\boldsymbol{u}_{\delta, i} \times \boldsymbol{u}_{r, i}\right) \cdot \boldsymbol{u}_{r^{\prime}, j}}{\sin \psi_{i, j}} \\
& =\frac{\boldsymbol{u}_{\alpha, i} \cdot \boldsymbol{u}_{r^{\prime}, j}}{\sin \psi_{i, j}}=\frac{\cos \delta_{j}^{\prime} \sin \left(\alpha_{j}^{\prime}-\alpha_{i}\right)}{\sin \psi_{i, j}} ; \\
\sin \gamma_{i} & =-\boldsymbol{n} \cdot \boldsymbol{u}_{\alpha, i}=-\frac{\left(\boldsymbol{u}_{r, i} \times \boldsymbol{u}_{r^{\prime}, j}\right) \cdot \boldsymbol{u}_{\alpha, i}}{\sin \psi_{i, j}}=-\frac{\left(\boldsymbol{u}_{\alpha, i} \times \boldsymbol{u}_{r, i}\right) \cdot \boldsymbol{u}_{r^{\prime}, j}}{\sin \psi_{i, j}} \\
& =\frac{\boldsymbol{u}_{\delta, i} \cdot \boldsymbol{u}_{r^{\prime}, j}}{\sin \psi_{i, j}}=\frac{\cos \delta_{i} \sin \delta_{j}^{\prime}-\sin \delta_{i} \cos \delta_{j}^{\prime} \cos \left(\alpha_{j}^{\prime}-\alpha_{i}\right)}{\sin \psi_{i, j}} .
\end{aligned}
$$

Similarly,

$$
\begin{aligned}
\cos \gamma_{j}^{\prime} & =\frac{\cos \delta_{i} \sin \left(\alpha_{j}^{\prime}-\alpha_{i}\right)}{\sin \psi_{i, j}} \quad \text { and } \\
\sin \gamma_{j}^{\prime}= & \frac{\cos \delta_{i} \sin \delta_{j}^{\prime} \cos \left(\alpha_{j}^{\prime}-\alpha_{i}\right)-\sin \delta_{i} \cos \delta_{j}^{\prime}}{\sin \psi_{i, j}}
\end{aligned}
$$

Let

$\boldsymbol{t}:=\boldsymbol{n} \times \boldsymbol{u}_{r, i}$

$\left(\approx \boldsymbol{n} \times \boldsymbol{u}_{r^{\prime}, j}\right.$ since $M_{i}$ and $M_{j}^{\prime}$ are close): vector $\boldsymbol{t}$ is a unit vector tangent in $M_{i}$ to the minor arc of great circle going from $M_{i}$ to $M_{j}^{\prime}$. Project the sphere on the plane $\left(M_{i}, \boldsymbol{t}, \boldsymbol{n}\right)$ tangent to the sphere in $M_{i}$ (the specific projection does not matter since we consider only $K^{\prime}$-sources in the neighborhood of $\left.M_{i}\right)$. We have

$$
\boldsymbol{r}_{i, j} \approx \psi_{i, j} \boldsymbol{t}
$$

and the basis $(\boldsymbol{t}, \boldsymbol{n})$ is obtained from $\left(\boldsymbol{u}_{a}, \boldsymbol{u}_{b}\right)$ by a $(\beta+\gamma-\pi / 2)$ counterclockwise rotation around $+\boldsymbol{u}_{r}$, so,

$$
\left(\Gamma_{i}\right)_{(t, n)}=\operatorname{Rot}^{\top}\left(\beta_{i}+\gamma_{i}-\pi / 2\right) \cdot \operatorname{Diag}\left(a_{i}^{2}, b_{i}^{2}\right) \cdot \operatorname{Rot}\left(\beta_{i}+\gamma_{i}-\pi / 2\right),
$$

$$
\left(\Gamma_{j}^{\prime}\right)_{(t, \boldsymbol{n})}=\operatorname{Rot}^{\top}\left(\beta_{j}^{\prime}+\gamma_{j}^{\prime}-\pi / 2\right) \cdot \operatorname{Diag}\left(a_{j}^{\prime 2}, b_{j}^{\prime 2}\right) \cdot \operatorname{Rot}\left(\beta_{j}^{\prime}+\gamma_{j}^{\prime}-\pi / 2\right)
$$

\section{A.2.2. Case of unknown positional uncertainties}

If the positional uncertainty on $M_{i}$ is unknown, we may model it with $\left(\Gamma_{i}\right)_{(t, n)}=\sigma^{2} \operatorname{Diag}(1,1)$, using the same $\sigma$ for all $K$ sources, and derive an estimate of $\stackrel{\circ}{\sigma}:=\sigma$ by maximizing the likelihood to observe the distribution of $K$ - and $K^{\prime}$-sources (see Sects. 3.2 and 4.2). For a galaxy, however, the positional uncertainty on its center is likely to increase with its size. If the position angle $\theta_{i}$ (counted eastward from the north) and the major 
and minor diameters $D_{i}$ and $d_{i}$ of the best-fitting ellipse of some isophote are known for $M_{i}$ (for instance, parameters PA, $D_{25}$ and $d_{25}:=D_{25} / R_{25}$ taken from the RC3 catalog (de Vaucouleurs et al. 1991) or HYPERLEDA (Paturel et al. 2003)), we may model the positional uncertainty with

$$
\begin{aligned}
\left(\Gamma_{i}\right)_{(\boldsymbol{t}, \boldsymbol{n})=} & \operatorname{Rot}^{\top}\left(\theta_{i}+\gamma_{i}-\pi / 2\right) \cdot \operatorname{Diag}\left(\sigma^{2}+\left[v D_{i}\right]^{2}, \sigma^{2}+\left[v d_{i}\right]^{2}\right) \\
& \cdot \operatorname{Rot}\left(\theta_{i}+\gamma_{i}-\pi / 2\right) \\
= & \sigma^{2} \operatorname{Diag}(1,1)+v^{2} \operatorname{Rot}^{\top}\left(\theta_{i}+\gamma_{i}-\pi / 2\right) \\
& \cdot \operatorname{Diag}\left(D_{i}^{2}, d_{i}^{2}\right) \cdot \operatorname{Rot}\left(\theta_{i}+\gamma_{i}-\pi / 2\right),
\end{aligned}
$$

and derive estimates of $\stackrel{\circ}{\sigma}:=\sigma$ and $\stackrel{\circ}{\nu}:=v$ from the likelihood. Such a technique might indeed be used to estimate the accuracy of coordinates in some catalog (see Paturel \& Petit 1999 for another method).

If the positional uncertainty on $M_{j}^{\prime}$ is unknown too, we can also put

$$
\begin{aligned}
& \left(\Gamma_{j}^{\prime}\right)_{(t, \boldsymbol{n})}=\sigma^{\prime 2} \operatorname{Diag}(1,1) \\
& +v^{\prime 2} \operatorname{Rot}^{\top}\left(\theta_{j}^{\prime}+\gamma_{j}^{\prime}-\pi / 2\right) \cdot \operatorname{Diag}\left(D_{i}^{2}, d_{i}^{2}\right) \cdot \operatorname{Rot}\left(\theta_{j}^{\prime}+\gamma_{j}^{\prime}-\pi / 2\right)
\end{aligned}
$$

with the same $\sigma^{\prime}$ and $v^{\prime}$ for all $K^{\prime}$-sources. As $\gamma_{j}^{\prime}+\theta_{j}^{\prime}=\gamma_{i}+\theta_{i}$, only estimates of $\stackrel{\circ}{\sigma}:=\left(\sigma^{2}+\sigma^{\prime 2}\right)^{1 / 2}$ and $\stackrel{\circ}{v}:=\left(v^{2}+v^{\prime 2}\right)^{1 / 2}$ may be obtained ${ }^{12}$ by maximizing the likelihood, not the values of $\sigma$, $\sigma^{\prime}, v$ or $v^{\prime}$ themselves.

\section{A.2.3. Possibly different true positions}

A similar technique can be applied if the true centers of $K$-sources and of their counterparts in $K^{\prime}$ sometimes differ. This might be useful in particular when associating galaxies from an optical catalog and from a ultraviolet or far-infrared one, because, while the optical is dominated by smoothly-distributed evolved stellar populations, the ultraviolet and the far-infrared mainly trace star-forming regions. Observations of galaxies (e.g., Kuchinski et al. 2000) have indeed shown that galaxies are very patchy in the ultraviolet, and the same has been observed in the far-infrared.

Since the angular distance between the true centers should increase with the size of the galaxy, we might model this as

$\boldsymbol{r}_{0, j}^{\prime}-\boldsymbol{r}_{0, i} \sim G_{2}\left(\mathbf{0}, \Gamma_{0, i}\right), \quad$ where

$\left(\Gamma_{0, i}\right)_{(t, \boldsymbol{n})}=v_{0}^{2} \operatorname{Rot}^{\top}\left(\theta_{i}+\gamma_{i}-\pi / 2\right) \cdot \operatorname{Diag}\left(D_{i}^{2}, d_{i}^{2}\right) \cdot \operatorname{Rot}\left(\theta_{i}+\gamma_{i}-\pi / 2\right)$.

We then have

$\boldsymbol{r}_{i, j} \sim G_{2}\left(\mathbf{0}, \Gamma_{i, j}\right), \quad$ with $\Gamma_{i, j}:=\Gamma_{i}+\Gamma_{j}^{\prime}+\Gamma_{0, i}$.
Once again, if $\sigma, \sigma^{\prime}, v, v^{\prime}$ and $v_{0}$ are unknown, only $\stackrel{\circ}{\sigma}:=\left(\sigma^{2}+\right.$ $\left.\sigma^{\prime 2}\right)^{1 / 2}$ and $\stackrel{\circ}{v}:=\left(v^{2}+v^{\prime 2}+v_{0}^{2}\right)^{1 / 2}$ may be estimated through likelihood maximization.

Acknowledgements. The initial phase of this work took place at the NASA/ Goddard Space Flight Center, under the supervision of Eli Dwek, and was supported by the National Research Council through the Resident Research Associateship Program. We acknowledge them sincerely. We also thank Stéphane Colombi for the discussions we had on the properties of maximum likelihood estimators.

\section{References}

Bartlett, J. G., \& Egret, D. 1998, in New Horizons from Multi-Wavelength Sky Surveys, eds. B. J. McLean, D. A. Golombek, J. J. E. Hayes, \& H. E. Payne, IAU Symp., 179, 437

Bauer, F. E., Condon, J. J., Thuan, T. X., \& Broderick, J. J. 2000, ApJS, 129, 547

Benn, C. R. 1983, The Observatory, 103, 150

Brand, K., Brown, M. J. I., Dey, A., et al. 2006, ApJ, 641, 140

Budavári, T., \& Szalay, A. S. 2008, ApJ, 679, 301

Condon, J. J., Balonek, T. J., \& Jauncey, D. L. 1975, AJ, 80, 887

Condon, J. J., Anderson, E., \& Broderick, J. J. 1995, AJ, 109, 2318

de Ruiter, H. R., Arp, H. C., \& Willis, A. G. 1977, A\&AS, 28, 211

de Vaucouleurs, G., \& Head, C. 1978, ApJS, 36, 439

de Vaucouleurs, G., de Vaucouleurs, A., Corwin, Jr., H. G., et al. 1991, Third Reference Catalogue of Bright Galaxies (New York: Springer)

Fioc, M. 2014, AsPECTs: code documentation and complements [arXiv: 1404 . 4224]

Fleuren, S., Sutherland, W., Dunne, L., et al. 2012, MNRAS, 423, 2407

Haakonsen, C. B., \& Rutledge, R. E. 2009, ApJS, 184, 138

Kendall, M., \& Stuart, A. 1979, The advanced theory of statistics. Vol. 2: Inference and relationship (London: Griffin)

Kent, J. T. 1982, J. Roy. Stat. Soc. Ser. B, Stat. Methodol., 44, 71

Kim, S., Wardlow, J. L., Cooray, A., et al. 2012, ApJ, 756, 28

Kuchinski, L. E., Freedman, W. L., Madore, B. F., et al. 2000, ApJS, 131, 441

McAlpine, K., Smith, D. J. B., Jarvis, M. J., Bonfield, D. G., \& Fleuren, S. 2012, MNRAS, 423, 132

Moshir, M., Kopman, G., \& Conrow, T. A. O. 1992, IRAS Faint Source Survey, Explanatory supplement version 2 (IPAC)

Moshir, M., Copan, G., Conrow, T., et al. 1993, VizieR Online Data Catalog: II/156

Paturel, G., \& Petit, C. 1999, A\&A, 352, 431

Paturel, G., Bottinelli, L., \& Gouguenheim, L. 1995, Astrophys. Lett. Commun., 31, 13

Paturel, G., Petit, C., Prugniel, P., et al. 2003, VizieR Online Data Catalog: VII/237

Pineau, F.-X., Motch, C., Carrera, F., et al. 2011, A\&A, 527, A126

Press, W. H., Teukolsky, S. A., Vetterling, W. T., \& Flannery, B. P. 1992, Numerical recipes in Fortran. The art of scientific computing (Cambridge: University press)

Prestage, R. M., \& Peacock, J. A. 1983, MNRAS, 204, 355

Rohde, D. J., Gallagher, M. R., Drinkwater, M. J., \& Pimbblet, K. A. 2006, MNRAS, 369, 2

Roseboom, I. G., Oliver, S., Parkinson, D., \& Vaccari, M. 2009, MNRAS, 400, 1062

Rutledge, R. E., Brunner, R. J., Prince, T. A., \& Lonsdale, C. 2000, ApJS, 131, 335

Sutherland, W., \& Saunders, W. 1992, MNRAS, 259, 413

Vignali, C., Fiore, F., Comastri, A., et al. 2009, in Multi-wavelength Astronomy and Virtual Observatory (European Space Agency), eds. D. Baines, \& P. Osuna, 53

Wolfram, S. 1996, The Mathematica book (Cambridge University Press)

Wolstencroft, R. D., Savage, A., Clowes, R. G., et al. 1986, MNRAS, 223, 279

\footnotetext{
${ }^{12}$ However, as noticed by de Vaucouleurs \& Head (1978) in a different context, if three samples with unknown uncertainties $\sigma_{i}(i \in \llbracket 1,3 \rrbracket)$ are available and if the combined uncertainties $\sigma_{i, j}:=\left(\sigma_{i}^{2}+\sigma_{j}^{2}\right)^{1 / 2}$ may be estimated for all the pairs $(i, j)_{j \neq i} \in \llbracket 1,3 \rrbracket^{2}$, as in our case, then $\sigma_{i}$ may be determined for each sample. Paturel \& Petit (1999) used this technique to compute the accuracy of galaxy coordinates.
} 